\title{
Trends in Individual Income Growth: Measurement Methods and British Evidence
}

\author{
Stephen P. Jenkins \\ Department of Social Policy and STICERD \\ London School of Economics and Political Science, \\ London WC2A 2AE, U.K. \\ Email: s.jenkins@1se.ac.uk \\ joint with \\ Philippe Van Kerm \\ CEPS/INSTEAD \\ B.P. 48, L-4501 Differdange, Luxembourg \\ Email: philippe.vankerm@ceps.lu
}

February 11, 2011 


\begin{abstract}
Assessments of whose income growth is the greatest and whose is the smallest are typically based on comparisons of income changes for income groups (e.g. rich versus poor) or income values (e.g. quantiles). However, income group and quantile composition changes over time because of income mobility. To summarize patterns of income growth while also tracking the fortunes of the same individuals, a longitudinal perspective is required. For this case, we develop dominance conditions and summary indices for comparisons of distributions of individual income growth, together with associated methods of estimation and inference. Using these methods and data from the British Household Panel Survey, we study individual income growth for periods between 1991 and 2005. We show that income growth was significantly more pro-poor in the early years of the Labour government than in earlier Conservative years.
\end{abstract}

Keywords: individual income growth; pro-poor growth; progressive income growth;

income mobility; mobility profile; British Household Panel Survey

JEL Classification: D31; D63; I32

Funders: ESRC (MiSoC), Fonds National de la Recherche, Luxembourg 


\section{Non-Technical Summary}

Statistics about average growth in real income over a period of time such as a year are commonly used to assess social progress and receive widespread coverage in the media. However, looking at averages does not take account of the fact that some people's incomes grow more than that of others and, indeed, there may be losers as well as gainers. That is, there is a distribution within the population of changes in people's incomes over time. This paper considers how to summarize distributions of individual income growth and how to compare distributions for different periods of time. We go beyond the pictures provided by averages: our measures incorporate sensitivity to the extent to which income growth is greater for poor people than for rich people, i.e. the extent to which income growth is progressive or, put another way, pro-poor rather than pro-rich.

There is already research that looks at patterns of individual income growth. These assessments are typically based on comparisons of income changes for income groups (e.g. rich versus poor) or income values (e.g. quantiles). However, income group and quantile composition changes over time because of income mobility. The people who are poor this year are not the same people who were poor last year: some leave poverty and others enter. More generally, income groups throughout the income range change their composition over time. To summarize patterns of income growth while also tracking the fortunes of the same individuals, a longitudinal perspective is required. For this case, we show that patterns of income growth are usefully summarized using graphs called mobility profiles and cumulative profiles. Moreover, if a profile for period $A$ lies everywhere above the profile for period $B$, then income growth in period $A$ is preferred to that in period $B$. Progressivity-sensitive indices summarizing the amount of growth are also developed (with the average as a special case).

Using these methods and data from the British Household Panel Survey, we study individual income growth for periods between 1991 and 2005. Our substantive results complement existing examinations of patterns of income growth and distribution change under different governments, specifically the Labour government that took office in May 1997 and the Conservative government that it replaced. We show that in the early years of the Labour government (1998-2002), income growth was more progressive than in the preceding Conservative years (1992-1996 and 19951999), but it was not so later in Labour's term of office (2001-2005).

There are ready explanations for the pro-poor nature of individual income growth in the 19982002 period. On the one hand, the economy was buoyant, with unemployment rates continuing to fall relatively rapidly from their early-1990s peak. On the other hand, the Labour government had an explicit anti-poverty agenda, unlike the preceding Conservative governments. Labour aimed to both raise employment rates by increasing the rewards to work relative to not working ('work first') and to help low-income working families ('making work pay'), thereby also reducing poverty rates especially child poverty rates. The introduction of Working Families Tax Credit in 1999 is an example of these policies. Why the progressive nature of income growth declined between 1998-2002 and 2001-2005 is an interesting question. We conjecture that the answer has to do with the slow-down in the economy from around 2000. 


\section{Public Economics Programme}

The Public Economics Programme was established in 2009. It is located within the Suntory and Toyota International Centres for Economics and Related Disciplines (STICERD) at the London School of Economics and Political Science. The programme is directed by Frank Cowell and Henrik Kleven. The Discussion Paper series is available free of charge. To subscribe to the PEP paper series, or for further information on the work of the Programme, please contact our Research Secretary, Leila Alberici on:

Telephone: UK+20 79556674

Fax: $\quad$ UK+20 79556951

Email: $\quad$ l.alberici@lse.ac.uk

Web site: http://sticerd.lse.ac.uk/PEP

(c) The Authors. All rights reserved. Short sections of text, not to exceed two paragraphs, may be quoted without explicit permission provided that full credit, including $\odot$ notice, is given to the source. 


\section{Introduction}

The income distribution in each year can be characterized as a Parade of Dwarfs and a few Giants (Pen, 1971). Each individual in the population is represented by a person who has a height proportional to the individual's income, and these representatives are lined up in order of height with the shortest at the front. Income growth over time corresponds to changes in the heights of Parade participants. There is typically a distribution of changes - there are winners and losers - a feature that is missed by a focus on the average growth rate. This paper considers how to summarize the distribution of individual income growth and to assess changes over time, and also provides empirical evidence for Britain on these topics.

The most commonly-used way of assessing the distribution of income growth is to compare incomes at a series of common points in the Parades for different years, e.g. at the deciles or vingtiles of each Parade. Growth incidence curves, graphs that summarize these calculations, are commonly used in development economics (Ravallion and Chen, 2003) and have also been used to summarize income growth in the UK (see e.g. Joyce et al., 2010, Chapter 3). There is also a substantial literature developing indices of the pro-poorness of income growth: see the review by Essama-Nssah and Lambert (2009).

The UK's official statistics on the personal income distribution, Households Below Average Income, provide information from this repeated cross-section perspective. For example, over the four years between fiscal years 1994/95 and 1998/99, income growth was relatively homogeneous across the middle half of the distribution, at a rate of around $10 \%$. At the tails, the experience was quite different: the $5^{\text {th }}$ percentile grew by $3.1 \%$ but the $95^{\text {th }}$ percentile grew by $11.6 \%$. By contrast, over the four years between fiscal years 1998/99 and 2002/03, income growth was greater the lower the percentile (with the exception of the $5^{\text {th }}$ percentile). The growth rate was almost $14 \%$ at the median and $17 \%$ at the $15^{\text {th }}$ percentile, but nearer $10 \%$ at the $75^{\text {th }}$ percentile and above. Overall inequality, as measured by the Gini coefficient, declined slightly. ${ }^{1}$

As a means of describing who has got better off or worse off, income growth statistics of the type just described are not fully satisfactory. Looking at the change in the income of the person at a specific quantile of Parade $A$ and the person at the same quantile of Parade $B$ ignores the fact that the persons concerned are not the same individuals. Over time, people change their position in the income Parade. Mobility means that the people who are poor this year are not the same people who were poor last year: some leave

\footnotetext{
${ }^{1}$ Authors' calculations from Department for Work and Pensions (2008, Table 2.1ts). The data are derived from the Family Resources Survey, an annual cross-sectional survey. The current HBAI series began in 1994/95, and the latest year for which statistics are available is 2008/09. The statistics cited in the text refer to periods that broadly correspond with those used in our own analysis reported later.
} 
poverty and others enter. More generally, income groups throughout the income range change their composition over time. To assess whether the individuals who are poor (or rich) this year are gainers or losers, one has to track the fortunes of individuals not the fortunes of income groups such as 'the poor' or 'the rich' whose composition may change from one year to the next. Longitudinal data are required, as it is only these that enable one to link the income of a specific individual in Parade $A$ with her income in Parade $B$, and hence calculate income growth for each individual.

In this paper, we develop methods that incorporate a longitudinal perspective. We provide a framework for social welfare evaluations of (changes in) the distribution of individual income growth, offering dominance results that are based on comparisons of mobility profiles and cumulative mobility profiles as well as scalar indices. For inference about changes over time in the distribution of income growth, we use bootstrap methods for dependent clustered data. The methods are used to analyze the distribution of income growth in Britain and how it has changed since the early 1990s.

Our substantive results complement existing examinations of patterns of income growth and distribution change under different governments, specifically the Labour government that took office in May 1997 and the Conservative government that it replaced. (Cf. the comparisons by Joyce et al. (2010) using the repeated cross-section perspective.) We show that in the early years of the Labour government income growth was more progressive than in the preceding Conservative years, but it was not so later in Labour's term of office.

We discuss potential explanations for the results in the concluding section but, for now, we note that statements of distributional goals have been made in terms of individual income growth as well as poverty and inequality reduction. Indeed, when asked in 2001 whether it was acceptable for the gap between rich and poor to get bigger, Prime Minister Tony Blair responded stating that 'the issue isn't in fact whether the very richest person ends up becoming richer. The issue is whether the poorest person is given the chance that they don't otherwise have', and 'the most important thing is to level up, not level down'. ${ }^{2}$ This is a statement supporting individual income growth as an income mobility concept. Positive growth is seen as good and negative growth as bad though, interestingly, Labour's Prime Minister expresses no strong support for greater income growth by the poorest persons relative to the richest persons. How to summarize and compare distributions of individual income growth when incomes may either rise or fall, while also incorporating a range of potential views about the social value of progressive income growth, is the subject of this paper.

\footnotetext{
${ }^{2}$ Interview on BBC Newsnight, 5 June 2001: transcript at http://news.bbc.co.uk/1/hi/ events/newsnight/1372220.stm.
} 
Section 2 develops methods for analysis of distributions of individual income growth from a longitudinal perspective, and contrasts them with conventional repeated crosssection methods. Our data, derived from the British Household Panel Survey (BHPS), including the measure of income, are described in Section 3. We develop methods of estimation and inference in Section 4. Our main empirical results are presented in Section 5. Section 6 investigates potential biases introduced by measurement error and sample ageing, and shows that accounting for them does not change our conclusions. (Details are provided in Appendices A and B.) Section 7 contains a summary and conclusions.

\section{Evaluating distributions of individual income growth}

This section considers how to derive welfare assessments of distributions of individual income growth. For evaluations of distributions of income levels, there are well-known results that demonstrate the equivalence between orderings of distributions according to increasing concave functions of incomes, configurations of quantile functions (Saposnik, 1981) and generalized Lorenz curves (Shorrocks, 1983), and orderings by classes of scalar indices of social welfare that are essentially 'inequality-adjusted' means (Jenkins, 1997, Gruen and Klasen, 2008). For comparisons of distributions of individual income growth, we develop relationships between social evaluation functions defined over bivariate income distributions, configurations of mobility profiles and cumulative mobility profiles, and orderings of classes of scalar indices of individual income growth that are essentially 'progressivity-adjusted' means.

\subsection{Progressivity-sensitive evaluations}

Most generally, a social evaluation function for patterns of individual income growth can be written $W(H)$ where $H$ is the joint distribution function of two positive random variables $X$ and $Y$ describing incomes in years $t$ and $t+\tau ; H(x, y)=\operatorname{Pr}[X \leq x, Y \leq y]$. This is also the starting point of much of the literature on the measurement of income mobility, reviewed by inter alia Maasoumi (1998), Fields and Ok (1999a), and Jenkins and Van Kerm (2009). An important lesson from that literature is that 'mobility is multi-faceted' (Fields, 2006, p. 123) and that '[t]he multidimensionality of the notion of mobility makes it impossible to devise a fundamental axiom that would unify all axiomatic enquiries that pertain to mobility measurement' (Fields and Ok, 1999b, p. 463). Different measures encapsulate different mobility concepts and can lead to different mobility orderings in practice (Fields et al., 2002, Checchi and Dardanoni, 2003). Individual income growth is one of the many mobility concepts: it is a form of 'income movement' to use the language of Fields and Ok (1999b). 
For assessments of distributions of individual income growth, we propose an evaluation function $W(H)$ with the following properties. The first is that $W(H)$ is the sum of individual-level income growth evaluations, $U(x, y ; H)$, each of which summarizes income growth for a person with income $x$ in year $t$ and $y$ in year $t+\tau$. $U(x, y ; H)$ may be positive, zero, or negative, since an individual's income may rise, stay the same, or fall over time. In other words, social evaluations are individualistic and additive, as commonly assumed in the income inequality and poverty measurement literatures. We allow $U$ to depend on the overall bivariate income distribution function $H$, so that the evaluation of a person's income growth may depend on the incomes of other individuals in the population.

A second property is replication invariance (Fields and Ok, 1999b, p. 463), again as used in the inequality and poverty measurement literatures: comparisons of two distributions are independent of the size of the populations. These first two properties imply that the overall social evaluation is a per-capita average:

$$
W(H)=\int_{z_{-}}^{z_{+}} \int_{z_{-}}^{z_{+}} U(r, s ; H) d H(r, s)
$$

where $\left[z_{-}, z_{+}\right]$is the support of $X$ and $Y$.

The third property concerns the specification of $U$. We propose that the individuallevel growth evaluation function for each person is given by the product of a component summarizing how much growth the person experiences (assessed in a manner common to all individuals) and a social weight that depends on the person's income in the base year. (This way of specifying an evaluation function follows Chakravarty (1984).) The aggregate evaluation function (1) can therefore be written as

$$
W(H)=\int_{z_{-}}^{z_{+}} \int_{z_{-}}^{z_{+}} \omega(r) \delta(r, s) d H(r, s)
$$

where the social weight is $\omega(x)>0$, and $\delta(x, y)$ is the 'growth distance function' common to all individuals. Assuming also that $\int \omega(x) d F_{X}=1$, where $F_{X}$ is the marginal distribution of base-year incomes, makes $W(H)$ a weighted average of individual income growth.

Additional properties of $W(H)$ arise from further assumptions about the two components of $U(\cdot)$. As we are describing income growth, we suppose that the growth distance function is directional. Fields and Ok (1999b, p. 460) define this to mean that $\delta(x, y)=-\delta(y, x)$ and also $\delta(x, \rho x)>\delta(x, x)$ for all $\rho>1$, where $\delta(\cdot)$ is a continuous function. We also introduce the normalization $\delta(x, x)=0$. These conditions ensure that positive income growth for an individual corresponds to a social improvement $(W(H)$ increases) and negative individual income growth corresponds to a reduction in $W(H)$, ceteris paribus. We also assume that the growth distance function respects either scale 
invariance or translation invariance: $\delta(\lambda x, \lambda y)=\delta(x, y)$ or $\delta(x+\eta, y+\eta)=\delta(x, y)$, respectively. In our empirical application, we focus on two specifications. The first is $\log (y)-\log (x)$ which is the case of proportionate income growth; the second is $y-x$ which is the case of absolute income growth. ${ }^{3}$ In conjunction with the other properties, scale invariance of $\delta(\cdot)$ implies scale invariance of $W(H)$, and translation invariance of $\delta(\cdot)$ implies translation invariance of $W(H)$.

The final property is a social preference for progressive income growth. This says that the impact on $W(H)$ of an increment to $\delta(\cdot)$ is greater (or no smaller), the lower the individual's income in the base year $(t): \omega^{\prime}(x) \leq 0$. Giving greater weight in the evaluation to initially-poorer individuals is consistent with, but not exactly the same as, a social preference for greater equality in final-year $(t+\tau)$ incomes than in base-year $(t)$ incomes. Greater equality in final-year incomes is guaranteed only if the pattern of income growth does not lead to re-ranking of individuals between the two years that is sufficiently large to offset the progressive income growth (Jenkins and Van Kerm, 2006). An important reference point is $\omega(x)=1$, for all $x$. This is the boundary case of neutrality towards differential income growth: $W(H)$ is then simply the population average of the individual-level growth statistics, which we write for brevity as $\bar{\delta}$. If $\delta(\lambda x, \lambda y)=\delta(x, y)$, it is the Fields and Ok (1999b) index of directional income movement with $c=1$.

An alternative but equivalent expression for $W(H)$ is useful for subsequent discussion. Since $H(r, s)=F_{Y \mid X=r}(s) \times F_{X}(r)$ where $F_{Y \mid X=x}$ is the cumulative distribution of finalyear incomes conditional on base-year income $x$, we can rewrite (2) as

$$
\begin{aligned}
W(H) & =\int_{z_{-}}^{z_{+}}\left(\int_{z_{-}}^{z_{+}} \omega(r) \delta(r, s) d F_{Y \mid X=r}(s)\right) d F_{X}(r) \\
& \equiv \int_{z_{-}}^{z_{+}} \omega(r) \mathrm{E}(\delta(r, y) \mid X=r) d F_{X}(r) \\
& =\int_{0}^{1} w(p) \mathrm{E}(\delta(x(p), y) \mid X=x(p)) d p
\end{aligned}
$$

where $\mathrm{E}(\delta(r, y ; H) \mid X=r)$ is the expected (average) income growth for individuals with a base-year income $r$. Equation (5) uses the change of variable $p=F_{X}(r)$, so $p \in[0,1]$ is the normalized rank in the base-year income distribution corresponding to income $r$, and $x(p)=F_{X}^{-1}(p)$ is the income corresponding to rank $p$ in the base-year distribution. The social weight is now written in terms of argument $p$ : it is $w(p)>0$, with $\int w(p) d p=1$.

\footnotetext{
${ }^{3}$ These are commonly-used ways of summarizing income growth, but not the only possibilities, e.g. a parametric class of scale-invariant growth distance functions is $\left(2^{\beta} / \beta\right)\left(y^{\beta}-x^{\beta}\right) /(x+y)^{\beta}, \beta \neq 0$, which is the 'arc percentage' divided by 100 in the case $\beta=1$. A translation-invariant class is $\left(2^{1-\gamma} / \gamma\right)[\exp (\gamma y)-$ $\exp (\gamma x)] /[\exp (\gamma x)+\exp (\gamma y)], \gamma \neq 0$. The limiting cases of $\beta=0$ and $\gamma=0$ are the proportionate and absolute growth distance functions cited in the text.
} 
For brevity, we refer to the conditional expectation as $m(p)$ and rewrite (5) as:

$$
W(H)=\int_{0}^{1} w(p) m(p) d p
$$

Thus, instead of writing the aggregate evaluation in terms of a weighted average of the individual evaluations, we can express it equivalently in terms of weighted averages of expectations of individual-level income growth conditional on position in the base-year income parade, $m(p)$. We use this alternative representation when discussing mobility profiles below. ${ }^{4}$

\subsection{Income mobility profiles and dominance relations}

We now show that unambiguous orderings of pairs of distributions of individual income growth according to social evaluation functions with the properties just discussed correspond to dominance defined in terms of mobility profiles and cumulative mobility profiles.

A mobility profile is the plot of $m(p)$ against $p$, given a definition of $\delta(\cdot) .^{5}$ The mobility profile reveals how income growth is distributed according to position in the base year income distribution. If income growth - assessed using $m(p)$ - is the same for everyone, the profile is horizontal. The mobility profile has negatively-sloped sections over the ranges of $p$ where individual income growth decreases as $p$ increases (progressive income growth); and the profile has positively-sloped sections over the ranges of $p$ where individual income growth increases as $p$ increases (regressive income growth). ${ }^{6}$

Cumulative mobility profiles are plots of the average income growth for people with an initial income at or below a given percentile $x(p)$ in the base-year distribution, i.e. among the poorest $100 p$ percent. That is, one plots

$$
\frac{1}{p} \int_{0}^{p} m(q) d q
$$

against $p \in[0,1]$. The resulting graph plots areas below the mobility profile - analogous to the way that a generalized Lorenz curve shows areas below a quantile function. The slope of the cumulative mobility profile may be positive or negative at different $p$ values. The cumulative profile's height at $p=1$ is $\bar{\delta}$.

We may now state two results concerning comparisons of distributions of income growth. For proofs (using integration by parts) and further discussion, see Van Kerm

\footnotetext{
${ }^{4}$ Most income mobility indices, regardless of mobility concept, can be written in the form shown by equations (3)-(6) (Jenkins and Van Kerm, 2009, Van Kerm, 2009).

${ }^{5}$ See Van Kerm $(2006,2009)$ for further discussion and illustrations. The mobility profile is an example of a fractile graph (Mahalanobis, 1960). Grimm (2007) independently proposed a similar device.

${ }^{6}$ Classical measurement error and transitory variability in incomes impart a negative slope to mobility profiles. We return to this regression-to-the-mean issue in Section 6.
} 
(2006, 2009). We consider comparisons between period $\mathcal{A}$ which refers to income growth between year $t$ and year $t+\tau$, and period $\mathcal{B}$ which refers to income growth between year $s$ and year $s+\tau$. Assessments are made using the evaluation functions described earlier: the comparison is of $W\left(H^{\mathcal{A}}\right)$ and $W\left(H^{\mathcal{B}}\right)$ for periods $\mathcal{A}$ and $\mathcal{B}$.

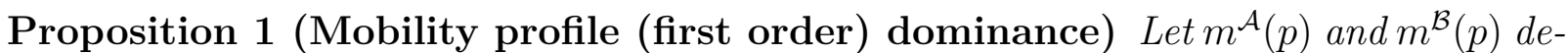
note the mobility profiles for periods $\mathcal{A}$ and $\mathcal{B}$ respectively. $m^{\mathcal{A}}(p) \geq m^{\mathcal{B}}(p) \forall p \in[0,1]$ if and only if $W\left(H^{\mathcal{A}}\right) \geq W\left(H^{\mathcal{B}}\right)$ for any $W$ with $w(p)>0$.

Proposition 2 (Cumulative mobility profile (second order) dominance) Let $C(p)=$ $\int_{0}^{p} m(q) d q . C^{\mathcal{A}}(p) \geq C^{\mathcal{B}}(p) \forall p \in[0,1]$ if and only if $W\left(H^{\mathcal{A}}\right) \geq W\left(H^{\mathcal{B}}\right)$ for any $W$ with $w(p)>0$ and $w^{\prime}(p) \leq 0$.

Mobility profile dominance states that finding the profile for period $\mathcal{A}$ is nowhere below and somewhere above the profile for period $\mathcal{B}$ is equivalent to the distribution of individual income growth for $\mathcal{A}$ being preferred to the distribution for $\mathcal{B}$ for any nonnegative social weight function $w(p)$. Cumulative mobility profile dominance states that finding the cumulative profile for period $\mathcal{A}$ is nowhere below and somewhere above the profile for period $\mathcal{B}$ is equivalent to the distribution of individual income growth for $\mathcal{A}$ being preferred to the distribution for $\mathcal{B}$ for any positive non-increasing weight function $w(p)$.

A corollary of the results is that profile dominance also provides orderings for evaluation functions specified in terms of incomes $(\omega(x))$ rather than normalized ranks $(w(p))$ : equations (3)-(5) show that $W(H)$ can be written in alternative ways, and the 'change of variable' employs a monotonic non-decreasing function $F_{X}$ which preserves the two dominance conditions.

These dominance results are fundamentally different from those used by Fields et al. (2002) and Chen (2009) to study income movement. Both they and we define profiles in terms of individual-level functions $(\delta(\cdot))$. The crucial difference is that they rank individuals from lowest to highest on the horizontal axis according to $\delta(\cdot)$ values, whereas the ranking in our case is by base-year income position. (Their graphical checks use Lorenz and generalized Lorenz curves of income movement statistics.) Income 'movement' (growth) in our case can be negative, not only zero or positive, and we build in a social preference for progressive growth.

Our mobility profile dominance results are related to those developed by Bourguignon (2010) also to study income growth patterns. The essential difference is that Bourguignon defines social welfare over the bivariate distribution of base- and final-year incomes, whereas we evaluate the distribution of income changes while incorporating a potential concern for progressive growth. While Bourguignon's specification of the social 
evaluation function is more general than ours in principle, his dominance results refer only to comparisons of bivariate distributions for periods $\mathcal{A}$ and $\mathcal{B}$ that have exactly the same base-year income distribution. This is a substantial restriction as it rules out analysis of changes in patterns of income growth over time or differences across countries. Base-year distributions that differ are the norm. In contrast, our methods are applicable to this case, as we illustrate later in the paper.

\subsection{Indices of progressivity-adjusted growth and return-to-progressivity}

Mobility profile dominance provides robust but only partial orderings of distributions of income growth with respect to the evaluation function $W$. Complete orderings by scalar indices that incorporate a social preference for progressive growth can be derived with further assumptions about the social weight function $w(\cdot)$. We refer to these as indices of 'progressivity-adjusted growth'.

In our analysis, we employ a class of single-parameter indices $W^{v}$ in which the social weight function is defined using the rank-dependent scheme that is implicit in the generalized Gini inequality index (Donaldson and Weymark, 1980, Yitzhaki, 1983):

$$
w(p)=v(1-p)^{v-1}
$$

where $v \geq 1$. For all $0<p<1, w(p)>0$, and $w^{\prime}(p) \leq 0$ as long as $v>1$, so the dominance results provide orderings for these indices. The larger that $v$ is, the faster the decrease in the social weight as $p$ increases, and hence the greater the preference for progressive income growth. If $v=2$, weights decrease linearly with $p$ from 2 to 0 ; if $1<v<2, w(p)$ is concave; and if $v>2, w(p)$ is convex. If $v=1, W^{v}=\bar{\delta}$.

$W^{v}$ reflects both levels of income growth and differences between individuals. There is also interest in summarizing the social return to the progressivity of individual income growth per se, i.e. the increase in social welfare that arises over and above the average growth experienced by the population as a whole. A natural index of the return to progressive growth is $G^{v}=W^{v}-\bar{\delta}$. This measure can be interpreted as the difference between observed average growth and equally-distributed-equivalent growth - the growth which, if received uniformly by each individual, would yield the same overall evaluation as the observed average growth were it also received uniformly. The more positive that $G^{v}$ is, the more progressive is income growth; negative values correspond to regressive income growth. 


\subsection{Longitudinal versus cross-sectional perspectives}

Mobility profiles and cumulative mobility profiles are 'non-anonymous' (Bourguignon, 2010, Grimm, 2007) versions of growth incidence curves and cumulative growth incidence curves. A growth incidence curve (Ravallion and Chen, 2003) is, in our notation, a plot of $\delta(x(p), y(p))$ against $p$ for $p \in[0,1]$, with $\delta(x, y):=100\left(\frac{y}{x}-1\right), x(p)=F_{t}^{-1}(p)$ and $y(p)=F_{t+\tau}^{-1}(p) \cdot{ }^{7}$ A cumulative growth incidence curve, labelled a poverty growth curve by Son (2004), shows the change in mean income among the poorest $p$ between two years, i.e. combinations of $\delta(\bar{x}(p), \bar{y}(p))$ and $p$, where $\bar{x}(p)$ and $\bar{y}(p)$ denote the mean income among the poorest $p$ at $t$ and $t+\tau$ respectively. Like a mobility profile and its cumulation, a growth incidence curve and its cumulation may have sections with positive or negative slopes, depending on the pattern of income growth across quantiles.

Growth incidence curves are based only on the two marginal distributions: income growth is summarized by changes in quantile values, and so each point on the curve refers to incomes for different individuals at $t$ and $t+\tau$. By contrast, the income mobility profile summarizes income changes for the same individual and depends on the bivariate distribution of income. Both the cumulative mobility profiles and the cumulative growth incidence curves show growth among the poorest $p$ (rather than growth at $p$ ) but, whereas the latter define the poorest $p$ separately according to each marginal distribution, cumulative mobility profiles define the poorest $p$ according to base-year incomes.

In our empirical application, we contrast the pictures describing patterns of income growth in Britain that are provided by conventional cross-sectional approach and our longitudinal approach.

\section{BHPS data: definition of income and subsamples}

We use data from waves 1-16 of the British Household Panel Survey (BHPS), corresponding to survey years 1991-2006. BHPS wave 1 is a nationally representative sample of the population of Great Britain living in private households in 1991. Original sample respondents (including each partner from a dissolved wave 1 partnership) have been followed over time and they, and their co-residents, were interviewed annually. Children in original sample households are also interviewed as adults when they reach the age of 16 years. The BHPS following rules ensure that the sample remains broadly representative of the population of Britain over time. ${ }^{8}$

\footnotetext{
${ }^{7}$ In our empirical work, we summarize proportionate growth using $\delta(x, y):=\log (y)-\log (x)$ rather than $\delta(x, y):=100\left(\frac{y}{x}-1\right)$ in order to provide a direct link with the mobility profile for proportionate growth. The two definitions result in growth incidence curves of almost identical shape.

${ }^{8}$ The BHPS is less representative of post-1991 immigrant groups. We do not use data from the extension samples for Scotland, Wales, and Northern Ireland that began in the late 1990s because it
} 
To assess whether patterns of income growth have changed over time, we report results for four periods, i.e. for four values of $t$ for pairs of years $t$ and $t+\tau$. The first period is 1992-1996 when there was a Conservative government led by John Major. We treat this period as a baseline against which we contrast three subsequent periods. The second period is 1995-1999. It began with a Conservative government and finished at the time the new Labour government (which entered office in May 1997) introduced major reforms to the benefit system, including tax credits, intending to help low-income families with children in particular. The third and fourth periods are 1998-2002 and 2001-2005, during which Labour was in power. Our periods are defined to differentiate between Conservative and Labour periods, though we are also constrained by the availability of income data (see below). ${ }^{9}$

Our analysis is descriptive in nature, aiming to shed light on the distribution of individual income growth and its progressivity, and how these have been changing over time. We do not seek to identify causal effects associated with particular policy reforms or particular governments. These caveats aside, our prior expectation was that income growth was more progressive under Labour than under the Conservatives. This prior was formed by the results of repeated cross-section analysis of income growth by, for example, Department for Work and Pensions (2008) and Joyce et al. (2010) and also by noting the anti-poverty aims of Labour's policy reforms at the end of the 1990s (more about these in Section 7). We are interested to see the extent to which our longitudinal perspective provides similar or different pictures to the repeated cross-section ones.

Our measure of an individual's income adheres as closely as possible to the 'before housing costs' net household income definition that is used in the UK's official income statistics, the Households Below Average Income series, cited in the Introduction. ${ }^{10}$

Income is the income of the household to which a person belongs, adjusted for differences in household size and composition using an equivalence scale, and expressed in constant prices. Specifically, income is 'current net household income', which is the sum across all household members of cash income from work, capital income, private and public transfers, minus direct taxes and occupational pension contributions. The income reference period is the month prior to the interview or the most recent relevant period,

would require use of complicated probability weighting schemes and the temporal coverage of the data is relatively short. For detailed discussion of the BHPS and its income data, see Jenkins (2010).

${ }^{9}$ Our conclusions are are not substantially affected by the specific choices of the periods for which results are reported. This is confirmed by calculations based on rolling four-year windows for the entire 1992-2005 period (results not reported for brevity).

${ }^{10}$ BHPS net income data files are an unofficial supplement to the official BHPS release, with 16 waves currently available. The data are documented by Levy and Jenkins (2008) and Jenkins (2010). Jenkins (2010) shows that BHPS cross-sectional income distributions closely match HBAI ones. For more details of the HBAI definition of income, see Department for Work and Pensions (2008). 
converted to a weekly equivalent pro rata. ${ }^{11}$ Total money income is equivalized using the modified-OECD equivalence scale, and expressed in January 2008 pounds using a 'before housing costs' price index series supplied by the Department for Work and Pensions.

In order to reduce the potential impact on our estimates of measurement error and transitory income fluctuations, each person's income is measured using a three-year longitudinal average: an individual's income measure for year $t$ is the arithmetic average of observed income for years $t-1, t$, and $t+1 .^{12}$ Longitudinal averaging of an individual's income smooths out errors and other transitory variation if measurement errors are classical (see Section 6). Also, to prevent outlier income values exerting undue influence, analysis throughout is based on samples from which incomes in the top $1 \%$ and the bottom $1 \%$ have been excluded (prior to taking three-year averages). Trimming of this kind is common in analysis of income dynamics: see e.g. Gottschalk and Moffitt (2009).

Given these definitions, our sample for estimation of growth over the period 1992-1996 is composed of respondents for whom income data are available for six survey years (1991, 1992, 1993, and 1995, 1996, 1997), and similarly for the other three time periods. The number of individuals in each subsample is: 6,088 (1992-1996), 6,130 (1995-1999), 5,789 (1998-2002), and 5,451 (2001-2005). Since the BHPS is an on-going panel survey, each of the four subsamples corresponding to the four periods has an overlapping membership. If there were no missing data on income, no attrition (or death) and no panel joiners (by coresidence or birth), each of the four sub-samples would comprise the same set of respondents observed at different points in time. Although sample overlap is incomplete in practice, there is dependence between the four subsamples and this needs to be taken account when calculating the standard errors of estimates. The bootstrap resampling algorithm that we use to address this is discussed in Section 4.

\section{Estimation and inference}

\subsection{Estimation}

Estimation of progressivity-adjusted growth indices, growth incidence curves and poverty growth curves is straightforward, because they involve standard estimators for concentration indices, quantiles, and incomplete means. We focus on estimation of mobility profiles.

\footnotetext{
${ }^{11}$ The use of a current rather than annual measure of income is standard in Britain. Böheim and Jenkins (2006) show that the BHPS current and annual income measures provide very similar estimates of distributional summary statistics.

${ }^{12}$ The use of three-year averaged income follows Gottschalk and Danziger (2001) and Jenkins and Van Kerm (2006). Use of single-year non-averaged incomes provides qualitatively similar results to those reported below.
} 
Income mobility profiles are examples of fractile graphs (Mahalanobis, 1960). Their estimation requires estimation of a conditional mean function non-parametrically, a problem for which a number of alternative estimators are available (Sen, 2005). We use robust locally weighted regression (LOESS) (Cleveland, 1979). LOESS involves determination of overlapping local neighbourhoods around each of a series of points $p$ spanning the range $[0,1]$, and then using data for the sample observations that fall in each neighbourhood to estimate mobility profile coordinates by the expected value of $\delta(x(p), y)$ at each $p$, denoted $\hat{m}(p)$. Local regression methods are particularly appropriate in this setting because of their well-known good behaviour near the boundaries of the support of the data, unlike running mean or standard Nadaraya-Watson kernel regression estimators.

Robust LOESS estimation is a two-stage procedure. At the first stage, the expected value at $p$ is derived from coefficients fitted using a weighted least squares regression in which the weights decline with the distance of observations from $p$ and are zero for observations outside of the neighbourhood:

$$
\hat{m}(p)=\hat{a}_{0} \quad \text { with } \quad\left(\hat{a}_{0}, \hat{a}_{1}\right)=\operatorname{argmin}_{a_{0}, a_{1}} \sum_{i} K\left(\frac{p_{i}-p}{h}\right)\left(\delta_{i}-a_{0}-a_{1}\left(p-p_{i}\right)\right)^{2}
$$

where $p_{i}=F_{t}\left(y_{i}\right)$ is the fractional rank of observation $i$ in the year $t$ income distribution and $\delta_{i}$ is a measure of individual income change (as described in Section 2) for observation $i$, namely $y_{i}-x_{i}$ or $\ln \left(y_{i}\right)-\ln \left(x_{i}\right) .{ }^{13}$ We follow Cleveland (1979) and adopt a tricube weight function for $K(z)=\left(1-|z|^{3}\right)^{3}$ if $|z|<1$ and 0 otherwise. (Using Epanechnikov kernel weights led to almost identical estimates.) We estimate $\hat{m}(p)$ at 25 equally spaced points over the range of $p(0.02,0.06, \ldots, 0.98)$. Local neighbourhoods are defined as $p \pm 0.075$ so that approximately 15 percent of each sample falls in each neighbourhood (i.e. $h=0.15) .{ }^{14}$

The second stage uses the first-stage estimator to derive individual weights that give less emphasis to outliers. That is, given an estimate of $\hat{m}^{0}(p)$ as in (9), a robustness weight for each observation is calculated as $k_{i}=Q\left(e_{i} / 6 \xi\right)$ where $Q(x)=\left(1-x^{2}\right)^{2}$ if $|x|<1$ and 0 otherwise, $e_{i}=\hat{m}^{0}\left(p_{i}\right)-\delta_{i}$ is the residual from the initially fitted curve, and $\xi$ is the sample median of $e_{i}{ }^{15}$ Given the weights, a new, robust, mobility profile estimate is calculated using the same formula as in (9) except that $K(\cdot)$ is replaced by $k_{i} K(\cdot)$. The second stage

\footnotetext{
${ }^{13}$ Observations with tied income values are assigned identical fractional ranks so that estimation results are independent of the sort order of the data. The mean of the fractional ranks is 0.5.

${ }^{14}$ LOESS differs from other local polynomial regression estimators (Fan and Gijbels, 1996) in the definition of the neighbourhood. LOESS uses a nearest neighbour approach that selects a fixed proportion of the sample around each grid point. Local polynomial regression typically uses a kernel-based neighbourhood of fixed width around grid points. In our application the two approaches are equivalent since the $\delta_{i}$ are regressed on ranks $p_{i}$ that are, by definition, uniformly distributed, and so a fixed fraction of the data falls into neighbourhoods of fixed width.

${ }^{15}$ Values for $\hat{m}^{0}\left(p_{i}\right)$ at all sample points $p_{i}$ are linearly interpolated from estimates of $\hat{m}^{0}(p)$ on the 25 grid points.
} 
can be repeated iteratively but the estimates we report are derived without additional iterations because preliminary analysis suggested that they had negligible impact. The robust LOESS procedure further minimizes the potential impact of outlier values (in addition to the data trimming and income smoothing mentioned earlier).

We estimate cumulative income mobility profiles by numerical integration of the robust LOESS estimates of the income mobility profiles using a trapezoidal rule (Press et al., 2007).

A bandwidth $h=0.15$ was chosen for estimation of $\hat{m}(p)$ after experimentation. This choice is a compromise between not under-smoothing the profiles and not over-smoothing the cumulative profiles (estimates of cumulative income mobility profiles are smoother than mobility profiles because of the cumulation process). For consistency's sake, we use the same bandwidth for all time periods. Estimates based on other bandwidths are available on request.

Sample weights are used to compute all estimates. Our four longitudinal samples are weighted using the BHPS cross-sectional enumerated individual weights of year $t+\tau$. We do not use the BHPS longitudinal weights because our analysis samples include sample joiners, and no longitudinal weights are provided for them.

\subsection{Resampling-based inference}

The sampling variability of our estimates, longitudinal and cross-sectional, is computed using a non-parametric block (panel) bootstrap procedure, as described by Cameron and Trivedi (2005, Chapter 11), which accounts for sample dependence that arises from the longitudinal nature of the data.

Resampling is from the sample of households interviewed in wave 1 of the BHPS. The full response history (including periods of non-participation if any) over BHPS waves 1-16 of all members of the selected households, plus their descendant split-off households and all respondents that later joined these households, is then selected to form a bootstrap replicate of the complete panel from wave 1 through 16 . To deal with survey design features (potentially small stratum sizes in particular), resampling from BHPS wave 1 households is done by cluster (primary sampling unit) within each sample stratum using the repeated half-sample bootstrap algorithm of Saigo et al. (2001). ${ }^{16}$ Let $\mathbf{X}^{b}$ denote one bootstrap replication $b$ of the full BHPS waves 1-16 sample, so constructed. To preserve the dependence and overlapping membership of the subsamples for the four periods analyzed, it is only in a second step that subsamples for 1992-1996, 1995-1999,

\footnotetext{
${ }^{16}$ The repeated half-sample bootstrap algorithm is also versatile as it has been demonstrated to lead to valid bootstrap inference with both smooth and non-smooth statistics (Saigo et al., 2001).
} 
1998-2002 and 2001-2005, are formed from each of the bootstrap replicate $\mathbf{X}^{b}$ of the full panel (according to the selection rules described above). Denote the replication $b$ subsample for period $(t, t+\tau)$ by $S_{t}\left(\mathbf{X}^{b}\right)$.

All of the statistics of interest described in Section 2 (including coordinates of the (cumulative) income mobility profiles or growth incidence curves at any $p$ ) are then estimated on each replicate subsample $S_{t}\left(\mathbf{X}^{b}\right)$ with $t \in\{1992,1995,1998,2001\}$ and $b \in$ $\{1,2, \ldots, B\}, B=999$. We denote any such estimate $\hat{\theta}_{t}^{b} \equiv \theta\left(S_{t}\left(\mathbf{X}^{b}\right)\right)$. We also calculate the difference between the $\hat{\theta}_{t}^{b}$ for each of the three later periods $(t, t+\tau)$ and that for the baseline period 1992-1996, $\hat{\Delta}_{t}^{b} \equiv \theta\left(S_{t}\left(\mathbf{X}^{b}\right)\right)-\theta\left(S_{1992}\left(\mathbf{X}^{b}\right)\right)$. By computing all estimates for the four sub-periods and the between-period difference statistics using the same sets of bootstrap replication, we capture the covariances between different measures across time.

Our $B$ replicates of $\hat{\theta}_{t}^{b}$ and $\hat{\Delta}_{t}^{b}$ are used to estimate the sampling error of our point estimates $\hat{\theta}_{t}$ and $\hat{\Delta}_{t}$, with the errors calculated as:

$$
\hat{s}\left(\hat{\theta}_{t}\right)=\sqrt{\frac{1}{B-1} \sum_{b=1}^{B}\left(\hat{\theta}_{t}^{b}-\bar{\theta}_{t}\right)^{2}}
$$

where $\bar{\theta}_{t}$ is the average of $\hat{\theta}_{t}^{b}$ over the $B$ replications. Variability bands are derived from the largest and smallest vingtiles of the $B$ replications: $\left[F^{-1}\left(0.05 ; \theta_{t}\right) ; F^{-1}\left(0.95 ; \theta_{t}\right)\right]$ where $F^{-1}\left(\alpha ; \theta_{t}\right)$ is the quantile $\alpha$ of the distribution of the $B$ bootstrap replications of $\hat{\theta}_{t}^{b}$. Standard errors and variability bands for $\hat{\Delta}_{t}$ are derived similarly.

We also use our bootstrap replications $\hat{\Delta}_{t}^{b}$ to evaluate the statistical significance of the sign of the observed changes over time. We do so by computing $\mathcal{P}\left(\hat{\Delta}_{t}\right)=\operatorname{Pr}\left[\operatorname{sgn}\left(\hat{\Delta}_{t}^{b}\right) \neq\right.$ $\left.\operatorname{sgn}\left(\hat{\Delta}_{t}\right)\right]$ which we evaluate as

$$
\mathcal{P}\left(\hat{\Delta}_{t}\right)=\frac{1}{B} \sum_{b=1}^{B} \mathbf{1}\left(\operatorname{sgn}\left(\hat{\Delta}_{t}^{b}\right) \neq \operatorname{sgn}\left(\hat{\Delta}_{t}\right)\right)
$$

where $\mathbf{1}(\cdot)$ evaluates to 1 if the expression in parentheses is true and 0 otherwise. $\mathcal{P}\left(\hat{\Delta}_{t}\right)$ gives the proportion of bootstrap replications for which the sign of $\hat{\Delta}_{t}^{b}$ is different from the sign observed in the point estimates $\hat{\Delta}_{t} \cdot \mathcal{P}\left(\hat{\Delta}_{t}\right)$ can be understood as the smallest $\alpha$ such that $(1-2 \alpha)$ variability bands for $\hat{\Delta}_{t}$ (as defined above) do not include zero. We interpret these as giving the probability of incorrectly inferring the sign of the difference from the sign of the point estimate of the difference.

We adopt similar procedures for assessing the statistical significance of the dominance relations between (cumulative) mobility profiles. We base our tests of mobility profile dominance of period $\mathcal{A}$ (years $t, t+\tau$ ) over the period $\mathcal{B}$ (years $s, s+\tau ; s>t$ ), on the 
statistic

$$
\operatorname{MP}(t, s)=\min _{p}\left[\hat{m}^{\mathcal{A}}(p)-\hat{m}^{\mathcal{B}}(p)\right]
$$

and tests of cumulative mobility profile dominance on the statistic

$$
\operatorname{CMP}(t, s)=\min _{p}\left[\int_{0}^{p} \hat{m}^{\mathcal{A}}(q) d q-\int_{0}^{p} \hat{m}^{\mathcal{B}}(q) d q\right]
$$

where $p$ takes the values $\{0.02,0.06, \ldots, 0.98\}$. Mobility profile dominance for period $\mathcal{A}$ over period $\mathcal{B}$ is established if $\operatorname{MP}(t, s) \geq 0$, and cumulative mobility profile dominance if $\operatorname{CMP}(t, s) \geq 0$.

The $\operatorname{MP}(t, s)$ and $\operatorname{CMP}(t, s)$ statistics are similar to the Kolmogorov-Smirnov statistics used in tests of stochastic dominance. Adapting the unrestricted bootstrap approach for stochastic dominance of Maasoumi and Heshmati (2000) to the analysis of mobility profile dominance, we claim observed dominance relations to be statistically significant at the $100(1-\alpha)$ percent confidence level if

$$
\left(\frac{1}{B} \sum_{b=1}^{B} \mathbf{1}\left(\mathrm{MP}^{b}(t, s)<0\right)\right)<\alpha,
$$

that is, if the proportion of bootstrap replications in which dominance is not observed (where it is observed in the original sample) is smaller than $\alpha \cdot{ }^{17}$ Cumulative mobility profile dominance is checked similarly.

\section{Patterns of individual income growth in Britain and their changes over time}

In this section, we provide a longitudinal perspective on individual income growth using the methods developed earlier, and compare the results with the pictures provided by growth incidence curves.

\subsection{Patterns of income growth: the longitudinal perspective}

Figure 1 shows income mobility profiles (left) and cumulative income mobility profiles (right) for the four time periods considered. The top two panels show income growth in absolute terms (the units are January 2008 pounds) and the bottom two panels show income growth in proportionate terms (the units are log January 2008 pounds). In all graphs, vertical dashed lines are used to demarcate the poorest and richest fifths of each

\footnotetext{
${ }^{17}$ We are in effect testing a null hypothesis of dominance against an alternative of non-dominance. Alternative approaches such as permutation tests or restricted bootstrap tests used in stochastic dominance analysis which allow tests of the null hypothesis of non-dominance are difficult to apply here given the complex dependence of the subsamples compared.
} 
base-year income distribution.

Mobility profiles are negatively-sloped, broadly speaking, regardless of whether an absolute or proportionate definition of growth is used, and for each of the four periods (albeit with a positive slope over some small ranges of $p$ ). That is, from a longitudinal perspective, the pattern of individual income growth is progressive: the lower the rank in the base-year distribution, the greater the expected income growth. Expected income growth, absolute or proportionate, is positive for the majority of individuals, but negative for individuals in the richest fifth in the base year.

The mobility profiles do not differ markedly across the four time periods. No curve lies completely above another at all values of $p$, so there is no first order dominance result. Taking all periods together, the estimates indicate that individuals starting in the poorest fifth experience an income increase of approximately 10\%-20\% over the subsequent four years, i.e. a little more than $£ 25$ per week. For those starting in the richest fifth, the corresponding change is around $-5 \%$.

Differences between periods in patterns of income growth are more clear cut when we consider cumulative income mobility profiles, especially when individual growth is measured in proportionate terms. The non-intersection of cumulative profiles suggests second-order dominance results, and unambiguous rankings by all members of our class of progressivity-adjusted growth measures. In the proportionate growth case, the ranking of the distributions of income growth for the periods is, from highest to lowest, 1998-2002, 1995-1999, 1992-1996, and 2001-2005. In the absolute growth case, the same ranking applies except that there is no dominance result concerning comparisons of 2001-2005 with 1995-1999 and 1992-1996: the cumulative profiles intersect in these cases.

These conclusions concerning dominance ignore sampling variability. Figures 2 and 3 show differences between the profile for 1992-1996 and each of the other three profiles, together with bootstrapped $90 \%$ variability bands. In each figure, the top panel refers to mobility profiles and the bottom panel to cumulative mobility profiles. Figure 2 refers to the absolute income growth case; Figure 3 refers to the proportionate income growth case. The other feature that is summarized in each of the graphs is the difference between periods in overall average individual income growth (the mean of the $\delta_{i}$ values, $\bar{\delta}$ ), shown as a horizontal dashed line. This provides an additional reference point for assessing change over time (see the discussion in Section 2).

Two features stand out from Figures 2 and 3. The first is the size of variability bands. They are sufficiently large that, with notable exceptions, differences in profiles are not statistically different from zero throughout the full range of $p$. They suggest that not all of the dominance results cited earlier are statistically significant. (We do not report all 
(a) Absolute mobility profiles

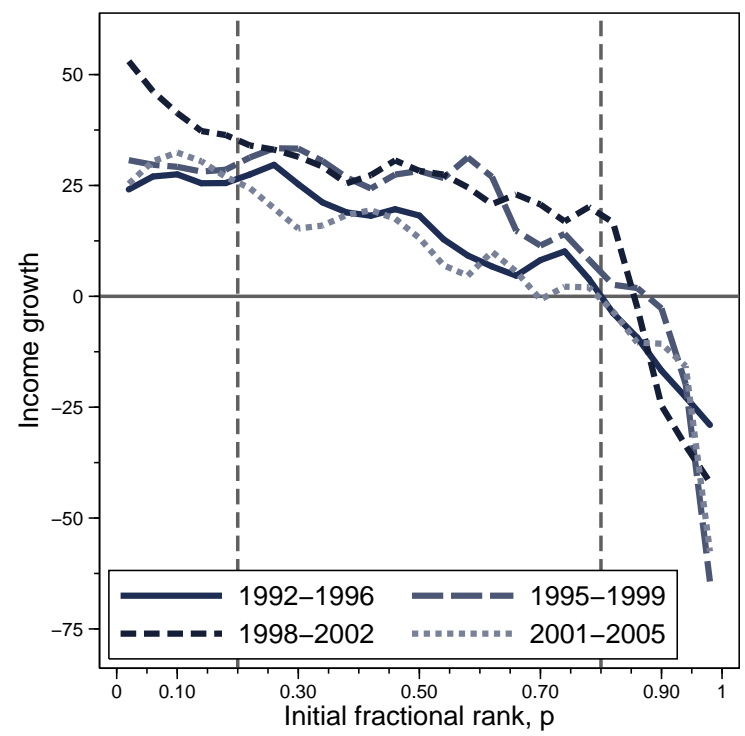

(c) Proportionate income mobility profiles

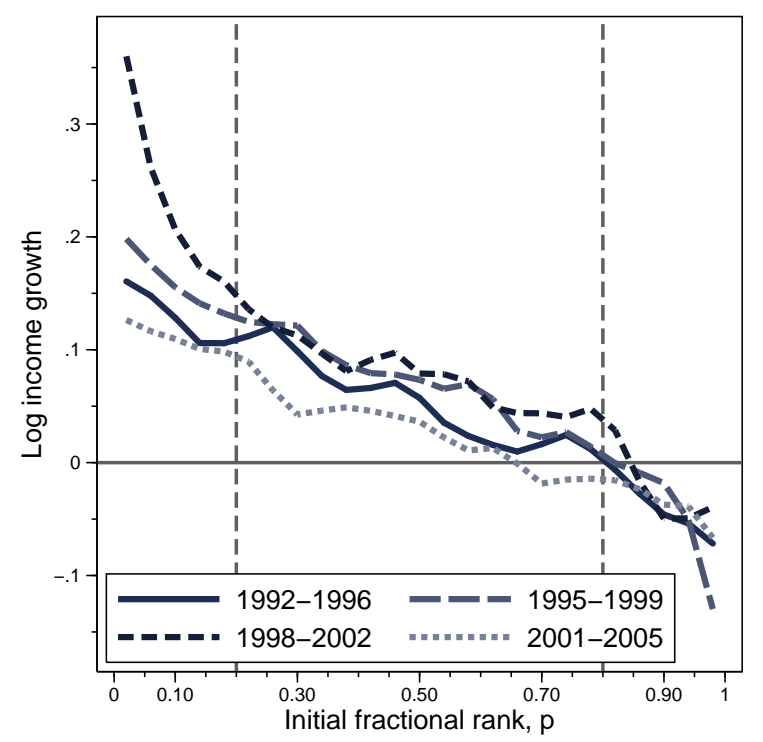

(b) Cumulative absolute mobility profiles

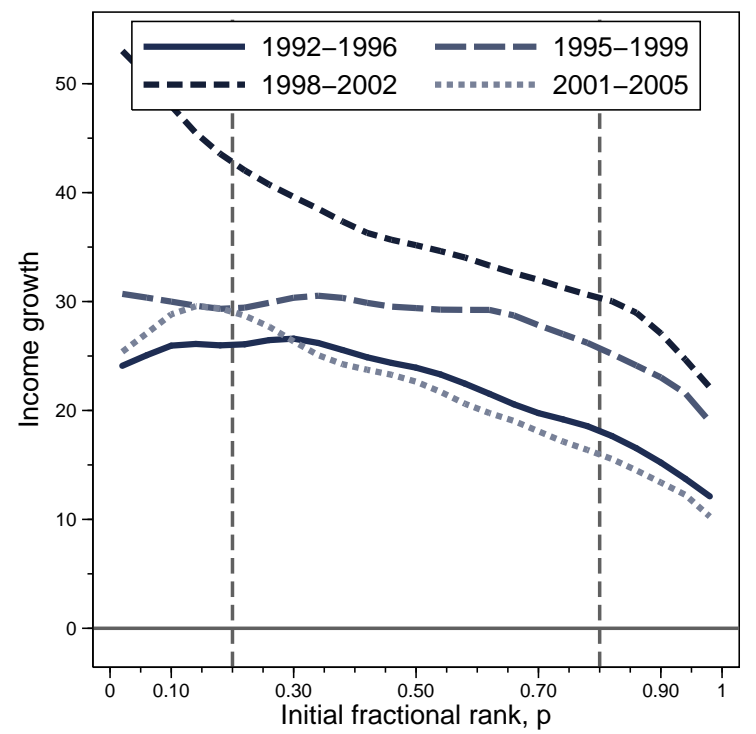

(d) Cumulative proportionate income mobility profiles

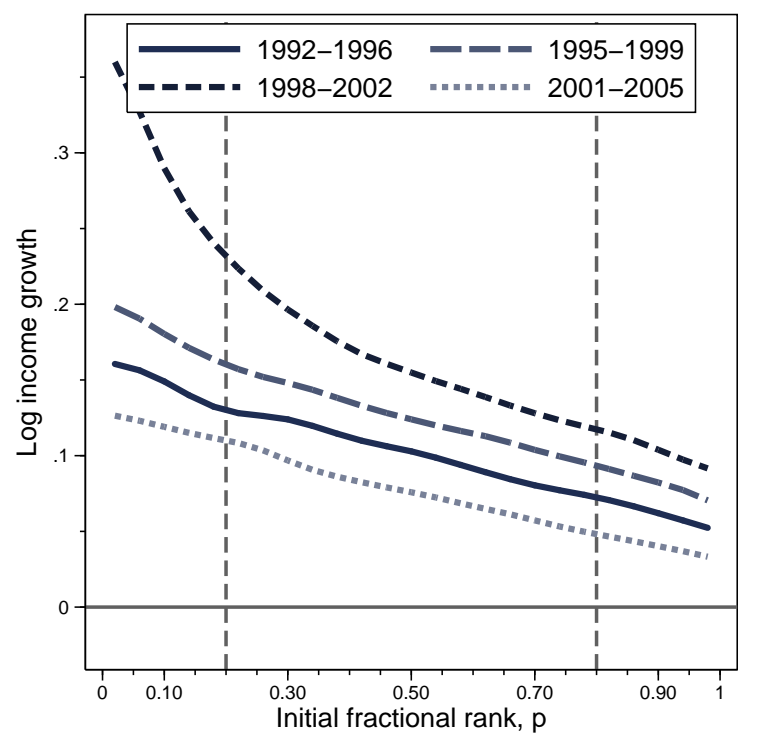

Figure 1. Income mobility profiles and cumulative mobility profiles

Note. Top panel shows absolute growth (change in real income); bottom panel shows proportionate growth (change in log real income). 
possible pairwise comparisons for brevity.)

The second feature concerns exceptions from the overall picture we have just described. On the one hand, these concern the comparisons involving 1998-2002 and the experience of the base-year poorest fifth in particular. Compared to 1992-1996, individual income growth is greater among the poorest fifth than among richer base-year income groups, and the variability bands lie above the zero growth reference point: there is first- and second-order dominance over this restricted range. On the other hand, the cumulative profile differences provide more clear cut results generally. There is statistically significant second-order dominance: the difference curves and associated variability bands lie above zero at all values of $p$. These patterns lead us to conclude that individual income growth was distinctively more progressive - and more pro-poor, specifically - in the 1998-2002 period than in 1992-1996. The comparisons between each of the other two periods and 1992-1996 do not stand out in this way. We can say that average growth was higher in the late 1990s period and lower in the early 2000s than in 1992-1996, but sampling variability prevents us from drawing definite conclusions about changes over time. 

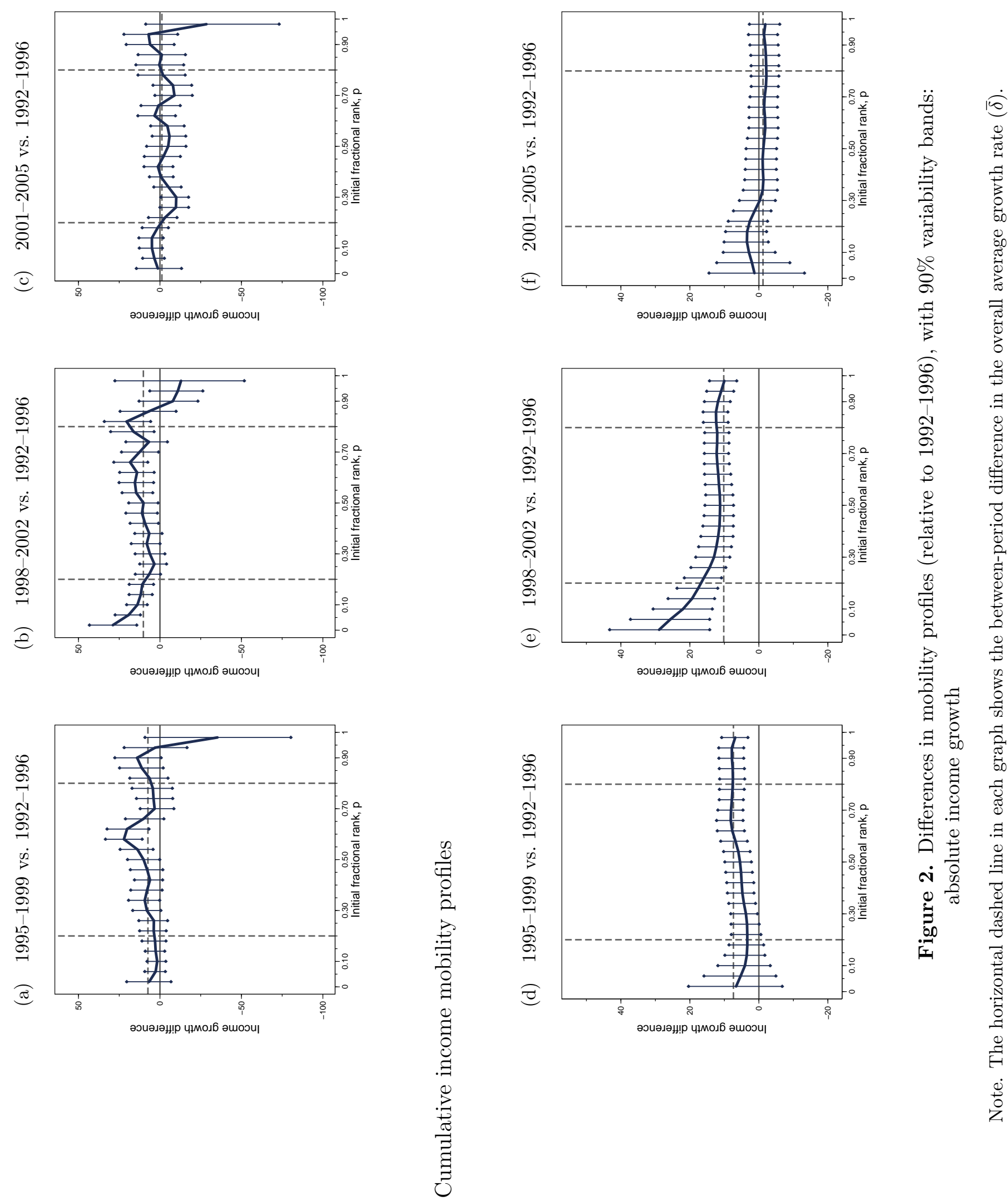

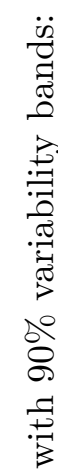



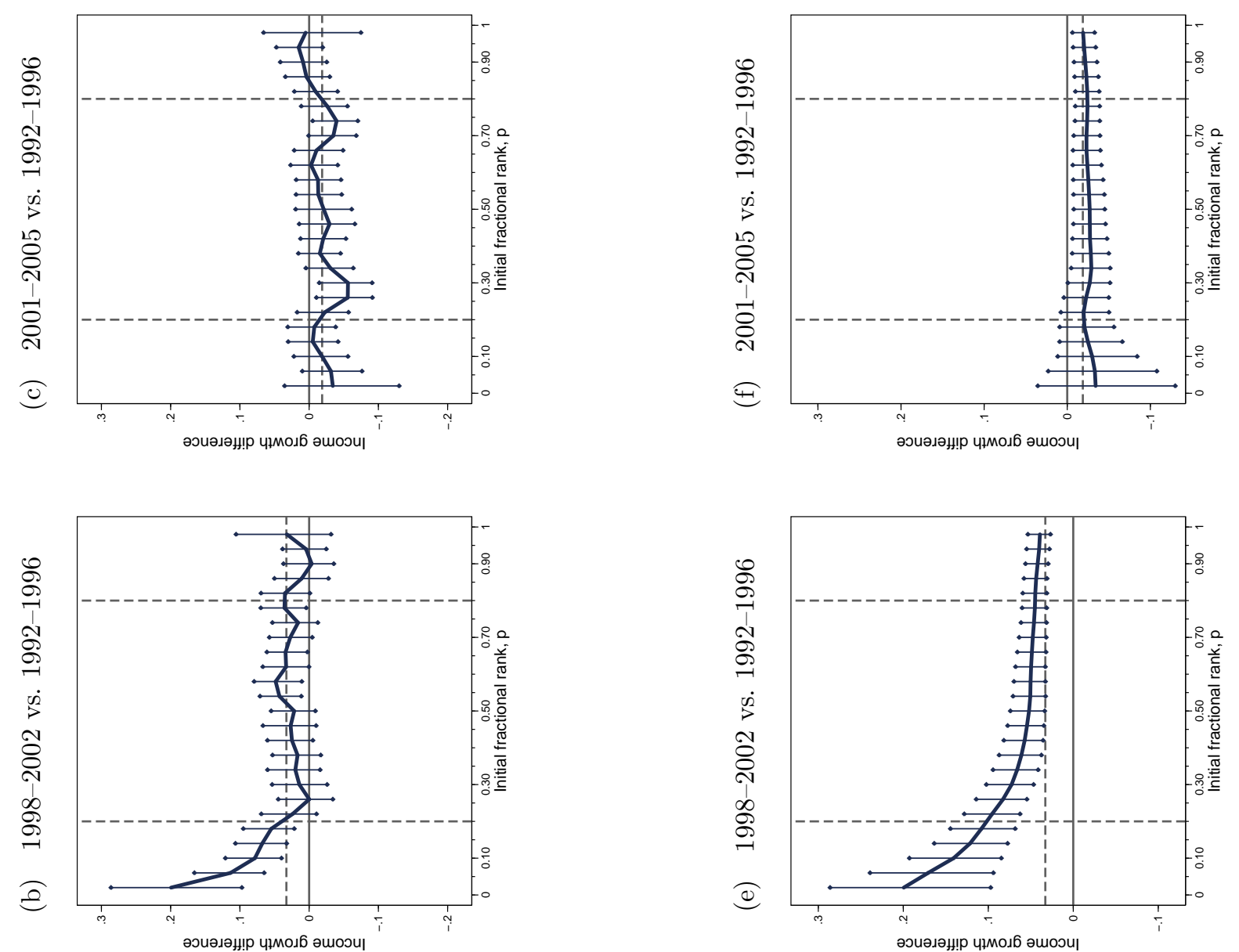

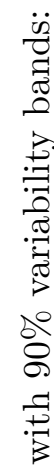



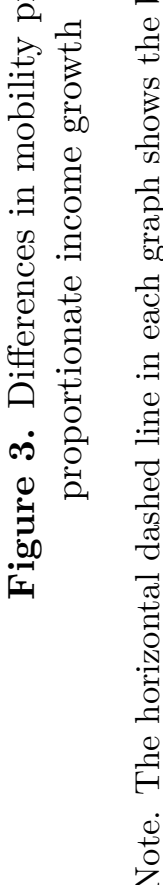

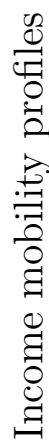
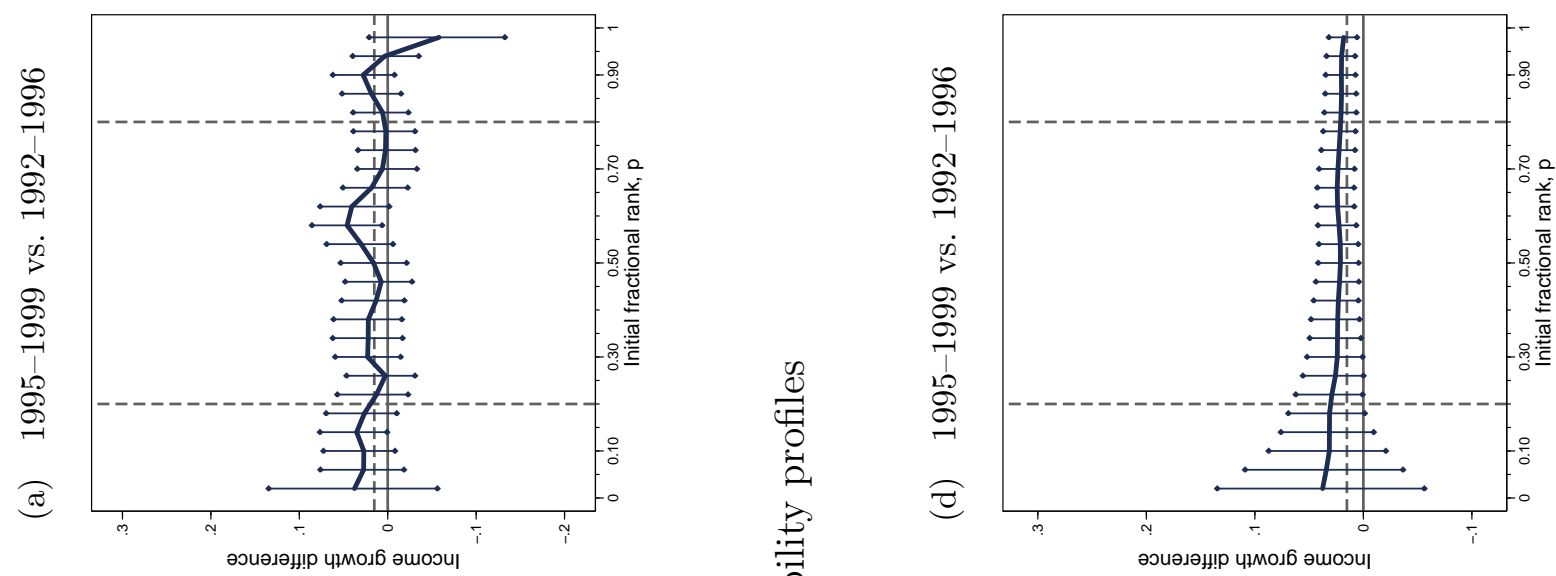
We now put numerical flesh on the patterns revealed so far. The upper panel of Table 1 reports, for each period, average income in the initial and final years, and average income growth over the period for the population as a whole. (The average growth estimates are estimates of the progressivity-adjusted growth index $W^{v}$ with $v=1$, i.e. $\bar{\delta}$.) The lower panel reports, for each statistic, the difference between the value for each of the three later periods and the 1992-1996 reference period. Corresponding estimates are shown in the middle and right-hand panels for individuals who were in the poorest fifth or the richest fifth in the relevant base year.

Table 1 confirms that average income growth for the population as a whole increased in each period, and in both absolute terms (from $\$ 15$ to $\$ 25$ ) and proportionate terms (from 5\% to 8\%) until 1998-2002. Income growth then fell to a level similar to that for the initial reference period; indeed, to a lower level in the proportionate growth case $(\$ 13$ or $3 \%$ ).

In every period, those who started in the poorest fifth had larger income gains than average (e.g. $\$ 33$ compared to $\$ 15$ in 1992-1996), and substantially larger gains than those who started in the richest fifth who experienced negative income growth in all periods (e.g. $-\$ 18$ in 1992-1996). The contrasts between the income change of the poorest and richest fifths were largest for the 1998-2002 period. This is a manifestation of the greater progressivity of income growth that we highlighted earlier. We summarize this progressivity using the progressivity-adjusted growth indices $G^{v}$ shortly.

The greater income gains for those starting in the bottom fifth are not sufficiently large to enable them to catch up with the richest fifth but the gap between them is reduced substantially. The ratio of the mean income of the richest fifth to the mean income of the poorest fifth in the base year ranges from 3.93 (425/108 in 1992) to $3.40(490 / 144$ in 2001) whereas the final-year ratios of mean income for the same two groups range from 2.87 (407/142 in 1996) to 2.60 (470/181 in 2005). Put differently, initial mean income differences between the richest fifth and the poorest fifth are reduced by approximately a quarter because income growth is progressive.

These calculations summarize income changes for groups defined by base-year income position, but income groups change their composition over time. Information about the prevalence of changes in income group composition is provided in the two columns labelled '\% move' in Table 1. The statistics show the proportion of individuals in a baseyear income group (poorest fifth; richest fifth) that are not in that group in the final year. There is an interesting symmetry: in each period, the fraction of individuals moving from the poorest fifth, or moving from the richest fifth, is the same - around one third. This is a relatively large proportion, and its size underscores the potential importance of taking a longitudinal perspective. 


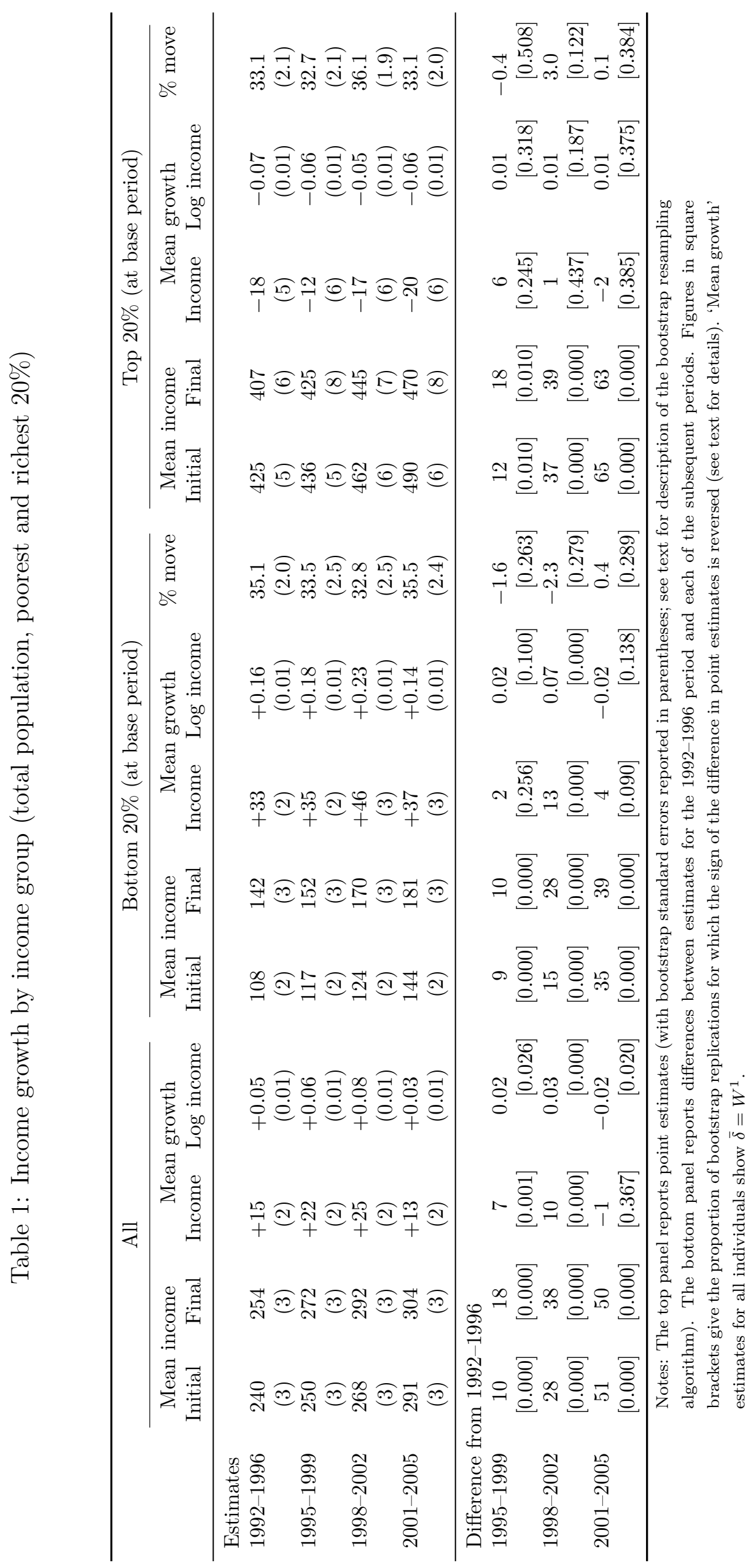


Income growth patterns are summarized further in Table 2 using our progressivityadjusted growth indices $\left(W^{v}\right)$ and return-to-progressivity indices $\left(G^{v}\right)$. Estimates for each period are shown in the top panel of the table, with the absolute growth measures to the left and proportionate growth measures to the right. We vary the progressivitysensitivity parameter $v$ from 2 (linear Gini-like weights) to 4 (placing greater weight on gains for initially-poorer individuals). It turns out that all the estimates of $W^{v}$ suggest the same trend over time. Progressivity-adjusted growth in every period was higher in each period than the preceding one, except in the final period when it fell. This was also the trend shown by the 'mean growth' estimates reported in Table 1 - these correspond to the case $W^{1}$.

Summaries of the differences in indices (relative to 1992-1996 values) are shown in the bottom panel of the table. The middle set of differences estimates, for 1998-2002, correspond to the case for which we found statistically significant second-order dominance. For this period, the difference in each index is large compared to the corresponding difference for other periods, and the associated ' $p$-value' is very small. Varying $v$ makes little difference to the estimates. For each $v$, income growth is about $£ 12$, or $5 \%$, and larger in 1998-2002 than in 1992-1996. The lack of variation in the difference estimates with changes in $v$ is also a feature of the comparisons for the other two periods. Observe, however, that the estimated differences involving 2001-2005 are near zero and lack statistical significance, whereas the corresponding estimates for 1995-1999 are positive, though consistently statistically significant only in the absolute growth case.

The larger estimates of $W^{v}$ for 1998-2002 partly reflect the greater average growth in this period reported in Table 1 and partly reflect greater growth progressivity. The estimates of $G^{v}$ isolate the latter component. The return-to-progressivity is positive for all periods, and significantly so. But the differences in $G^{v}$ between periods are less marked. It is only for 1998-2002 and for the proportionate income growth case that $G^{v}$ is significantly greater than for $1992-1996$. With $v=2$, the return to progressivity is some $2 \%$ larger in 1998-2002 than in 1992-1996, and thus accounts for about $40 \%$ of the total welfare difference calculated using $W^{2}$.

\subsection{Patterns of income growth: the repeated cross-section perspec- tive}

Figure 4 shows growth incidence curves (left) and cumulative growth incidence curves (right) for the same four time periods. For consistency, we use the longitudinal subsamples to calculate these. ${ }^{18}$ The graphs in the top panel show income growth in abso-

\footnotetext{
${ }^{18}$ Repeated cross-section estimates derived from cross-sectional subsamples are very similar to the estimates reported here.
} 


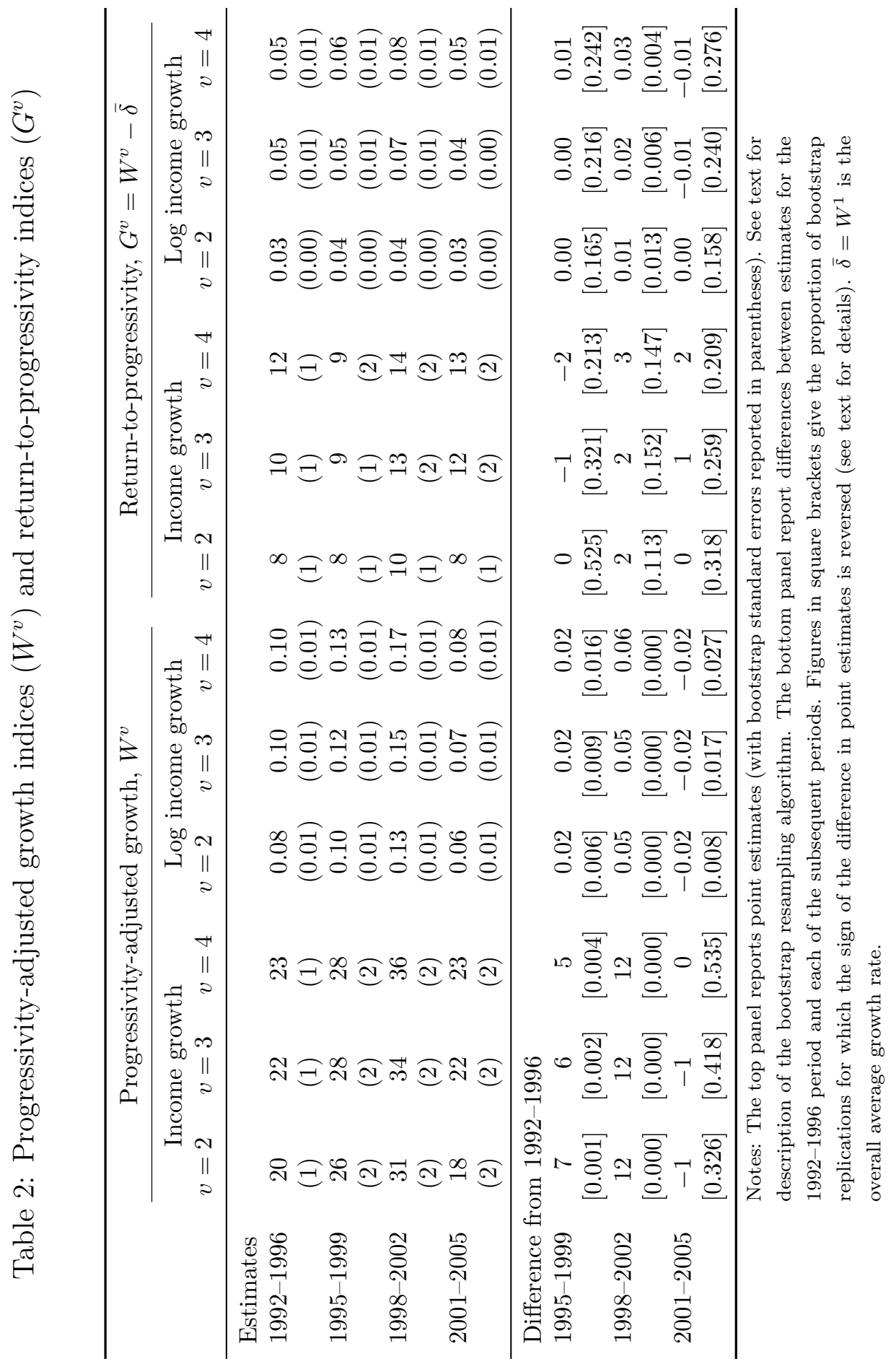


lute terms; those in the bottom panel show income growth in proportionate terms (i.e. growth incidence curves as conventionally defined). Figures 5 and 6 summarize differences between curves for 1992-1996 and those for the three later periods, together with bootstrapped variability bands. There are contrasts with and similarities between the patterns of income growth shown in these figures and those derived using a longitudinal perspective.

Broadly speaking, the mobility profiles shown in Figure 1 have a negative slope for both absolute and proportionate growth definitions, whereas the growth incidence curves shown in Figure 4 have a positive slope in the absolute growth case, and are flat or negatively-sloped in the proportionate growth case. Put another way, in the absolute growth case, the growth incidence curves indicate that the rich experience greater income growth than the poor over each of the four four-year periods, but the mobility profiles indicate that income increments are greater for the poor than the rich. How can these findings be reconciled? The answer is that, over each of the periods, most of the increase in income at the top percentiles is due to income gains by individuals who did not belong to the top income group in the base year but did so in the final year. Similarly, those at the bottom who experienced substantial income growth left their fellow poor behind, and were replaced in the final year by people who were not among the poorest initially. This is the marked extent of reranking in the income Parade over time that we documented earlier. $^{19}$

The principal similarities between the repeated cross-section and longitudinal perspectives are that differences in patterns of income growth are more easily distinguished from the cumulative graphs and, related, the orderings of the periods is similar. As with the mobility profiles, the growth incidence curves for different periods tend to overlap. With cumulation, the distinctive nature of the distribution of income growth over the 1998-2002 period in particular becomes strikingly apparent: the cumulative growth incidence curve for that period lies above the corresponding curves for the other three periods in both the absolute and proportionate growth cases. This is a second-order dominance result which also implies greater poverty reduction over the 1998-2002 period than the other ones, for all standard additive poverty indices and regardless of the generosity of the poverty line (Son, 2004). The pro-poor nature of income growth over the 1998-2002 period is particularly marked. In contrast, the curves for the other three periods intersect with each other.

Of course, these conclusions need modification when sampling variability is taken into account. Again there are parallels with the results from the longitudinal perspectives. Variability bands are relatively large and, in many of the pairwise comparisons of periods,

\footnotetext{
${ }^{19}$ For further details, see Jenkins (2011, Chapter 5).
} 
one cannot conclude that differences are statistically different from zero at all values of p. However, the differences between 1998-2002 and 1992-1996 are clearly positive and statistically significant, underlining the conclusions drawn in the last paragraph. In this case, the positive growth for the initially poorest fifth is also greater than the positive growth experienced by the population as a whole on average. 
(a) Absolute growth incidence curves

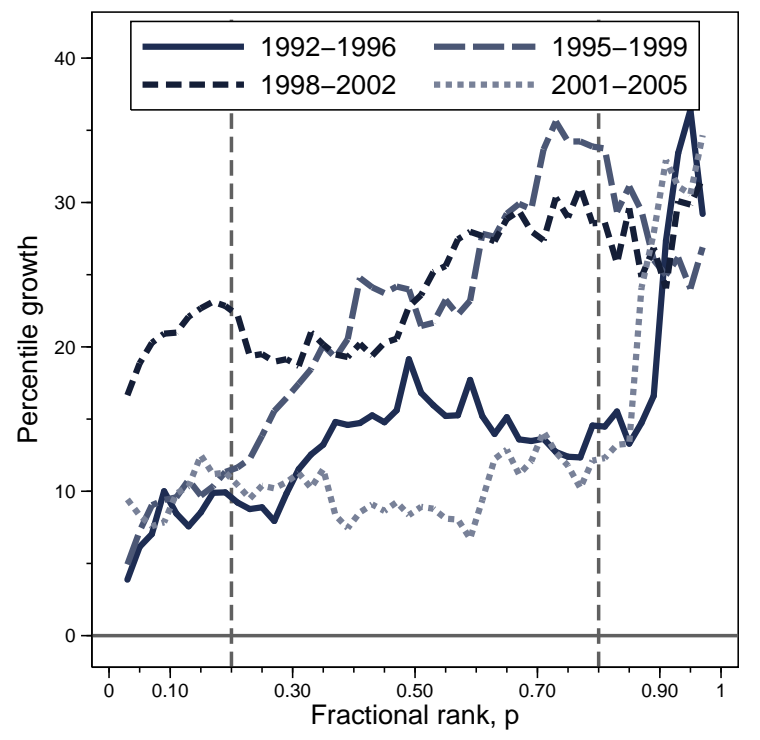

(c) Proportionate growth incidence curves

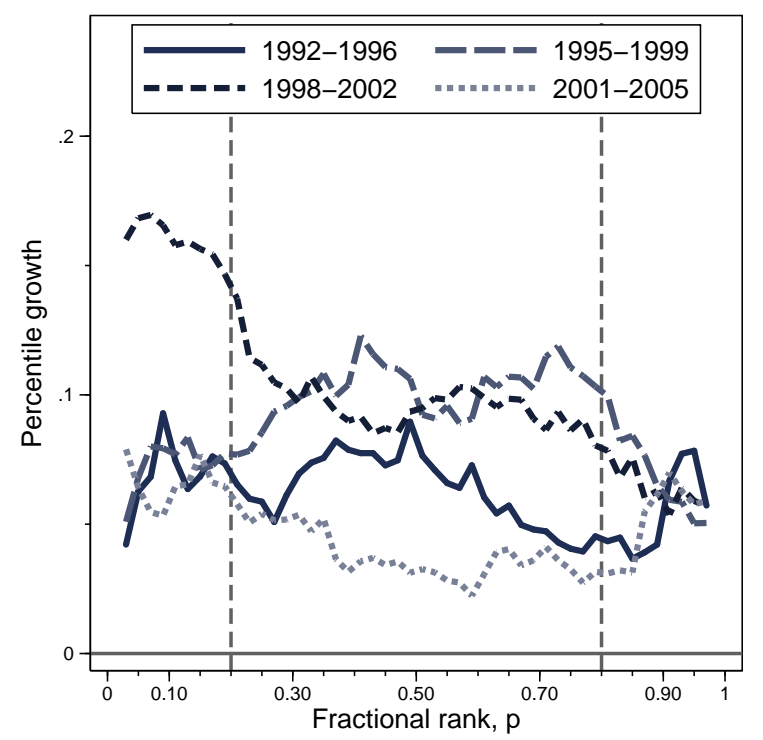

(b) Cumulative absolute growth incidence curves

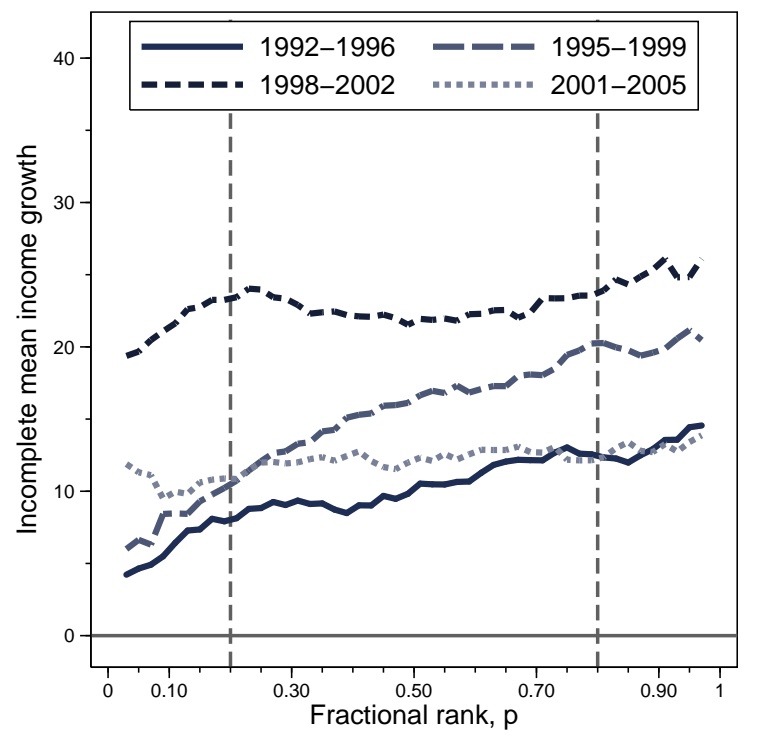

(d) Cumulative proportionate growth incidence curves

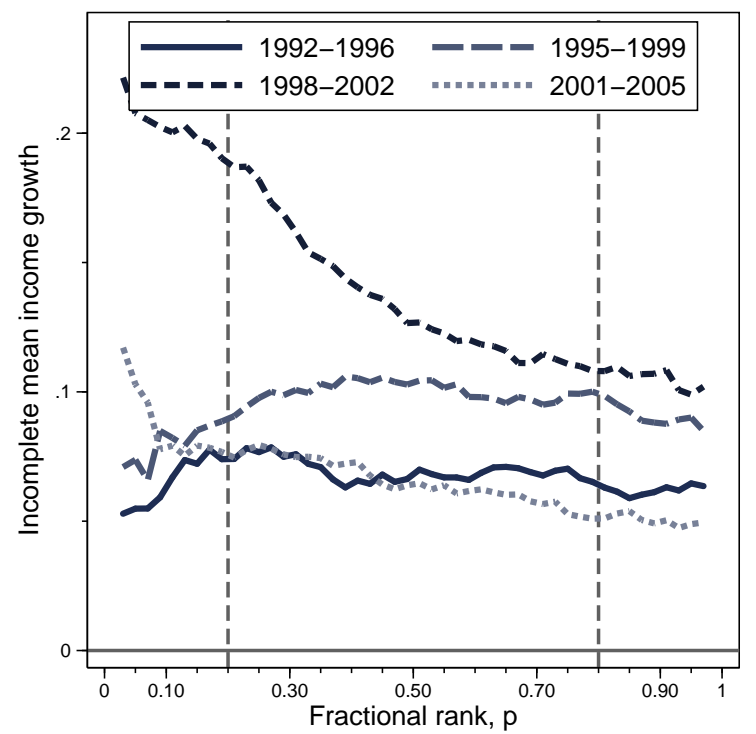

Figure 4. Growth incidence curves: income growth by percentile

Note. Top panel shows absolute growth (change in real income); bottom panel shows proportionate growth (change in log real income). 

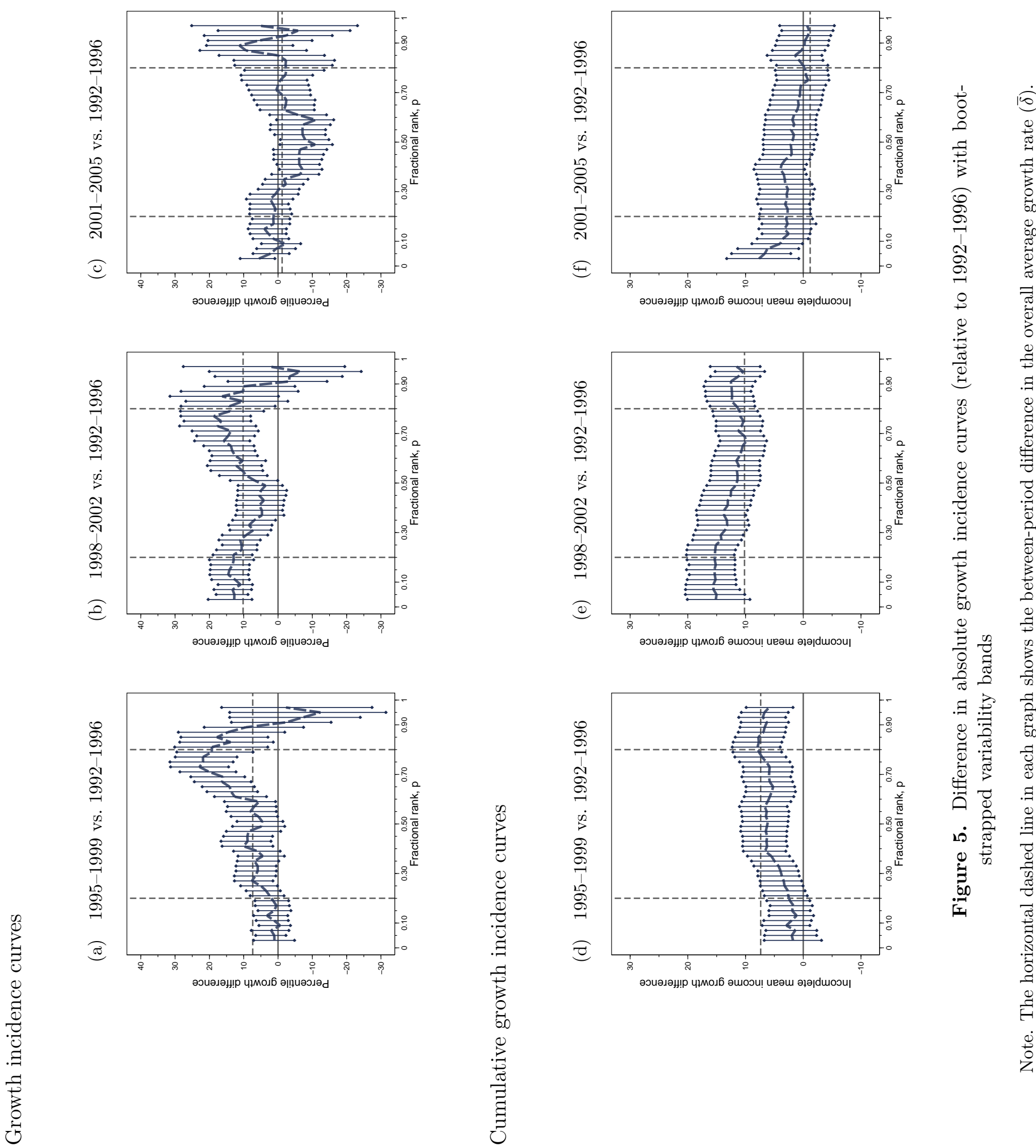

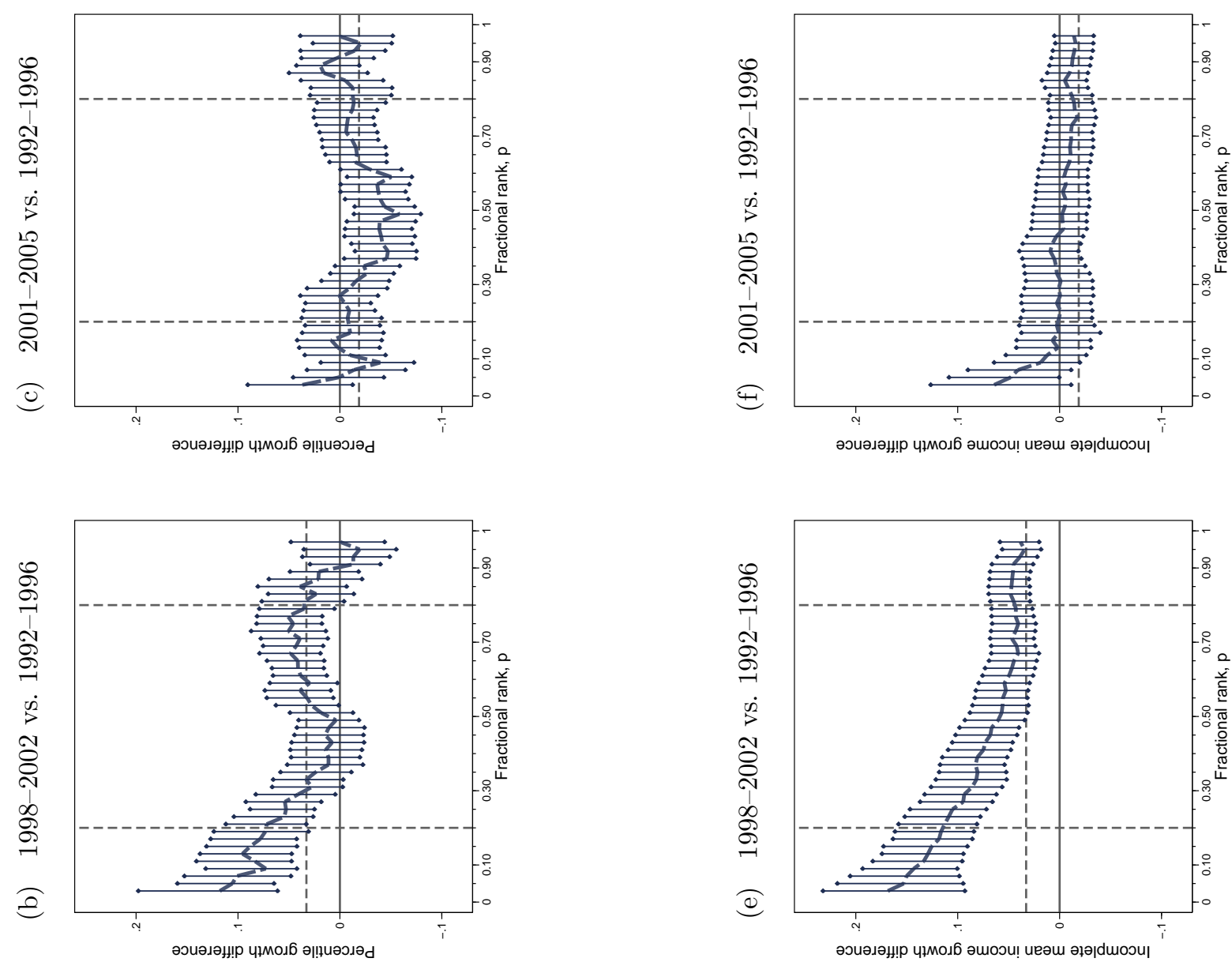

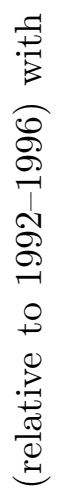
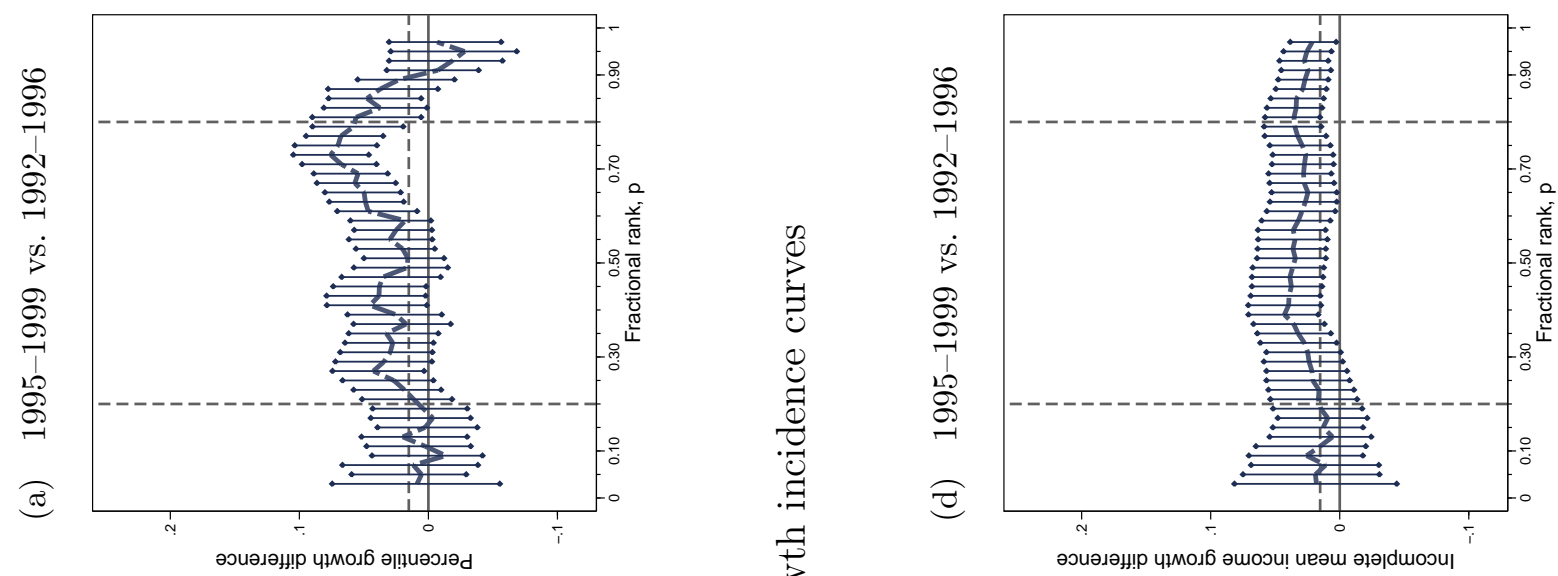

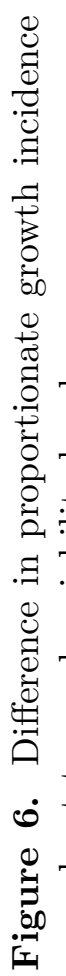

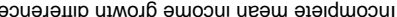




\section{Sensitivity analyses}

This section summarizes a number of analyses undertaken to investigate whether our conclusions about patterns of income growth and their temporal changes are sensitive to different treatments of measurement error or to changes across periods in the relationship between income and age.

\subsection{Regression to the mean and measurement error}

It might be argued that the negative slopes of our income mobility profiles are simply due to regression to the mean. The reasoning is that if there is classical measurement error (errors uncorrelated with the true value and over time), then the expected income increase for someone with a below-average income is positive and is negative for someone with above-average income. ${ }^{20}$ Hence, some of the observed progressivity in income growth evidenced in the income mobility profiles may be spurious. Our use of a three-year income average aims to reduce the impact of this problem by smoothing out measurement errors (and transitory variability) so that the economically substantive variations are better monitored. (As it happens, use of single-year incomes led to broadly similar findings.) One response to the regression to the mean argument is that it is not so relevant to comparisons of patterns over time. There is no change e.g. in the BHPS design that leads us to expect the nature of measurement error to have varied over time. A bias in the profile slope may be consistent with no bias in the estimated difference between profiles. Another response to the classical measurement error argument is that, in reality, measurement error may not be classical. Gottschalk and Huynh (2010) argue persuasively that factors such as mean reversion in errors offset biases arising from the variance inflation aspect of measurement error, so that bias in measures of mobility may be negligible. ${ }^{21}$

Validation data are not available to us to examine this issue, and so our investigations of the impact of measurement error employed two other strategies. The first is a procedure analogous to an instrumental variables (IV) approach to reduce the impact of measure-

\footnotetext{
${ }^{20}$ An analogy may help. If one rolls a standard die, the expected number of spots at any roll is 3.5 (the sum of the possible scores divided by six). If the first roll in fact produces a 1 , then the expected increase in the score when the die is rolled again is positive $(+2.5)$. By contrast, if a 6 comes up first, the expected gain at the second roll is negative $(-2.5)$. So, despite there being no association between the first and the second rolls (the die is fair), there is a correlation between the initial outcome and the change in outcome.

${ }^{21}$ Gottschalk and Huynh (2010) fit a measurement error model to linked survey and administrative record data on the earnings of US men. See also Dragoset and Fields (2006) who use the same data, but a larger collection of mobility measures, and find that qualitative findings are similar for administrative and survey data. Quantitative findings often differ more substantially but no systematic pattern is found. Fields et al. (2003) and Grimm (2007) use a calibrated version of a model similar to that of Gottschalk and Huynh (2010) to place bounds on the impact of measurement error on estimates of the correlation between incomes in two years.
} 
ment errors. The second focuses on samples with more reliably-measured incomes. We discuss these in turn.

Our 'IV' approach uses definitions of income and income growth that break the link between measurement error in base-year income level and in income growth, thereby offsetting any regression-to-the-mean driven by classical measurement error. We approximate individual base-year ranks, $F_{t}\left(y_{i t}\right)$, by $\tilde{F}_{t}\left(\tilde{y}_{i t}\right)$ where $\tilde{F}_{t}$ is the distribution function of $\tilde{y}_{i t}=y_{i t-1}+y_{i t+1}$. The idea is to use income lags and leads to approximate current period income and to derive base-year income rankings from this. We consider income change of the form $\delta\left(y_{i t}, y_{i t+\tau} ; H\right)$ where $y_{i s}$ is income for year $s$ (not a three-year smoothed average). These definitions imply that no data are used simultaneously to determine base-year rank and income change; it is as if people's ranks in the distribution of lag and lead incomes are used as instruments for their ranks in the distribution of current-year income.

Estimates employing the instrumented base-year ranks are presented in Appendix Figures A1, A2, and A3. The results are similar to those reported earlier. There is clear progressivity of income growth: mobility profiles remain negatively sloped. ${ }^{22}$ The estimated trends over time are also reassuringly similar to those reported earlier, including the distinctive pro-poor nature of the 1998-2002 period. All dominance results are maintained, with similar levels of statistical significance.

Our second strategy is to recalculate our estimates using subsamples for which we believe incomes are better measured. The first subsample is our main sample but excluding individuals belonging to a household in which at least one person is in full-time self-employment. The second subsample drops instead individuals belonging to households with income components imputed by BHPS staff because of item non-response. (We also employed a third subsample excluded both individuals in households with selfemployment and also those with imputed incomes, but subsample numbers were prohibitively small.) Self-employment income is notoriously difficult to capture reliably in surveys. Imputations lead to measurement error because of prediction error: to the extent that income is imputed in the final or initial year of a period, imputation is likely to lead to error in measurement of income change over time. One issue with the subsample strategy is that it can lead to substantial reductions in sample size. For example, dropping individuals from households with self-employed workers (in either the base or final year of each period) leads to reduction in sample size of about $20 \%-25 \%$. Dropping individuals in households with imputation of at least one major household income com-

\footnotetext{
${ }^{22}$ The curves are somewhat steeper than in our baseline estimates, but note that the income change reported here is not based on 3-year smoothing. Comparable estimates not based on 3-year smoothing and without proxying base yeear rank are substantially steeper, as expected.
} 
ponent reduces the sample by up to $75 \%-80 \%$. As a result, we increased the bandwidth used to derive the income mobility profile to $h=0.2$ for the 'no self-employed' subsample and to $h=0.3$ for subsamples excluding imputed data. Estimates for each of the two samples are shown in Appendix A.

Excluding self-employed households leads to no substantial change in results. Profiles are not markedly flatter (except for very high $p$ ). The main difference is that the growth distribution for 2001-2005 period is now ranked above that for the 1992-1996 period (using the absolute income change definition), but differences remain not statistically significant. When observations with imputed incomes are excluded, mobility profiles remain negatively-sloped but they become much flatter. Nevertheless, our general conclusions about trends remain valid, although statistical significance is now weaker: standard errors increase when sample sizes are substantially reduced.

In sum, the various checks suggest that our substantive conclusions are robust to the treatment of measurement error.

\subsection{Age-adjusted estimates}

There may be a concern that income growth patterns, and differences in them between periods, partly reflect the ageing of the sample. Incomes vary systematically with age (on average) and so, if the age composition of the sample changes over time, part of the change in the distribution of income growth may reflect this phenomenon. The BHPS design is intended to preserve representativeness and minimize the possibility of the sample ageing (through its following rules, and post-hoc using weights). However, to the extent that these intentions are not met and, more generally, to reassure ourselves that sample ageing is not driving our results, we have also computed our estimates using 'standardized' samples. These are samples in which the age structure of the 1992-1996 subsample is imposed on all three later subsamples. ${ }^{23}$

Standardisation is implemented by reweighting subsample observations so that the age distributions for each subsample are the same as the distribution in the first of the four periods. The reweighting factor for observation $i$ with age $a_{i}$ in the subsample for the later period beginning in year $s$ is defined as

$$
\pi_{i}(s)=\frac{\operatorname{Pr}\left(a=a_{i} \mid t=1992\right)}{\operatorname{Pr}\left(a=a_{i} \mid t=s\right)}
$$

where $\operatorname{Pr}\left(a=a_{i} \mid t=s\right)$ is the relative frequency of observations with age $a_{i}$ in the subsample for period $(s, s+\tau)$. Using Bayes' rule, the reweighting factor can be expressed

\footnotetext{
${ }^{23}$ This is similar to the technique of 'direct standardisation' that is commonly used in demography.
} 
equivalently as

$$
\pi_{i}(s)=\frac{\operatorname{Pr}\left(t=1992 \mid a=a_{i}\right)}{\operatorname{Pr}\left(t=s \mid a=a_{i}\right)} \frac{\operatorname{Pr}(t=s)}{\operatorname{Pr}(t=1992)}
$$

where $\operatorname{Pr}(t=s)$ is the proportion of observations from period $(s, s+\tau)$ in a pooled sample of 1992-1996 and period $(s, s+\tau)$ observations, and $\operatorname{Pr}\left(t=s \mid a=a_{i}\right)$ is the proportion of period $(s, s+\tau)$ observations in this pooled sample given age equal $a_{i}$. The reweighting factors are then multiplied by the sample weights and applied in all computations for periods other than 1992-1996. We estimated reweighting factors using expression (16) with the conditional probabilities computed from a probit model in which the covariates represent a linear spline in age with knots placed at ages 5, 12, 25, 35, 45, 60 , and 70 .

The estimates are displayed in Appendix Figures B1 and B2. They show that differences over time for age-standardized estimates are virtually identical to the nonstandardized estimates that we presented earlier.

\section{Discussion}

The thesis of this paper is that analysis of the pattern of income growth, and its changes over time, should employ longitudinal perspectives to complement conventional repeated cross-section approaches. It is of interest to know how the incomes of specific individuals change over time, not only how the incomes associated with Parade positions (income 'slots') change over time. There is not a one-to-one relationship between the two perspectives because there is substantial reranking of individuals in the income distribution over time. We have therefore developed longitudinal methods - methods that relate configurations of mobility and cumulative mobility profiles to unanimous orderings by aggregate measures of progressivity-adjusted income growth. For statistical inference, we have applied bootstrap resampling methods that take account of the dependent clustered samples that are inherent in this type of analysis.

Our estimates for four four-year periods in recent British history suggest that, from a longitudinal perspective, income growth is generally progressive. Over any particular period, income growth is greater for those with lower incomes in the base-year distribution. This is not an artefact of measurement error or sample ageing. This pattern is quite different from the picture provided by growth incidence curves (a repeated cross-section perspective): broadly speaking, they suggest that income growth over a four-year period has been typically been regressive in terms of absolute income changes though more distributionally neutral in terms of proportionate income changes.

Within this general picture, the results for the 1998-2002 Labour period stand out 
as distinctively pro-poor, however, relative to both the two preceding four-year periods (when there was a Conservative government) and relative to the subsequent four-year period (when Labour continued to form the government).

Our finding concerning the progressive nature of income growth during the early years of Labour's administration is fully consistent with earlier analysis which has taken a repeated cross-section perspective almost exclusively. What we have done is to analyze income growth patterns in greater detail, triangulating cross-sectional and longitudinal perspectives, and giving greater attention to the measures used for assessment and issues such as statistical inference (which turn out to be important) and measurement error and sample ageing (which do not).

There are ready explanations for the pro-poor nature of income changes in the 19982002 period. On the one hand, the economy was buoyant, with unemployment rates continuing to fall relatively rapidly from their early-1990s peak. On the other hand, the Labour government had an explicit anti-poverty agenda, unlike the preceding Conservative governments. Labour aimed to both raise employment rates by increasing the rewards to work relative to not working ('work first') and to help low-income working families ('making work pay'), thereby also reducing poverty rates especially child poverty rates. The principal programmes directed at these ends were Working Families Tax Credit (WFTC), introduced in October 1999, providing means-tested support to low income working families that was more generous than its predecessor (Family Credit). (WFTC was itself replaced by the Working Tax Credit and Child Tax Credit programmes from April 2003.) There were other changes during the period that raised support for families with children, notably increases in universal Child Benefit, and increases in the child allowances in Income Support, the social assistance benefit for those not in paid work. A national minimum wage was introduced in April 1999. To reduce pensioner poverty, a means-tested minimum income guarantee for poor pensioners was introduced in April 1999. ${ }^{24}$ Labour's policies had significant impacts on the income distribution according to microsimulation analysis. For example, Sefton and Sutherland (2005) compare estimated incomes in 2004/05 under the 2004/05 tax-benefit rules and estimated incomes in 2004/05 had policies remained as they were in 1997. Their overall conclusion is that 'the impact of the tax and benefit policies introduced since 1997 imply a significant redistribution towards those on low incomes, compared with what would have happened if the old system has simply been adjusted for inflation' (p. 244).

Why the progressive nature of income growth declined between 1998-2002 and 20012005 is an interesting question. To us, the smoking gun is the slow-down in the economy

\footnotetext{
${ }^{24}$ See inter alia Brewer and Shephard (2004) and Bennett and Millar (2005) for further details of the policies introduced by the Labour government.
} 
between the two periods. The rate of decline in the unemployment rate levelled off around 2000 and remained at around 5\%, and the annual rate of GDP growth fell from around $4 \%$ to $3 \%$ (Jenkins, 2011, Chapter 3), and wage growth for full-time employees was much lower (Sefton et al., 2009, Table 2.1). That is, there was a reduction in the capacity to deliver additional redistribution. Developing more detailed answers is beyond the scope of this paper, but the link between redistributive capacity and the state of the economy is an interesting topic for further research.

Another important topic for future research is closer examination of patterns of income growth for subgroups within the population such as lone parent and other families with children, pensioners, and so on, and relating these to developments at the population level. This would provide more descriptive evidence about the identity of the gainers and losers within a given period. It would also assist development of narratives about policy reform effects. For instance, Labour reforms were primarily directly at families with children and pensioners and so, if it is policy reform rather than the state of the economy that drives changes in patterns of income growth, one would expect to see differential income improvements among these groups in particular. The methods developed in this paper are not ideal for analysis of this kind. What is required are measures of progressivitysensitive growth that are decomposable by population subgroup. Our measures are not decomposable because the measures are contingent on base-year ranks. (The Gini coefficient of inequality is not exactly decomposable as a weighted sum of subgroup Ginis for the same reason.)

There is a more fundamental issue related to subgroup decomposability that also needs to be addressed in the longitudinal context. That is, people move between population subgroups over time (e.g. with family formation and dissolution); subgroup membership changes as well as income group membership. Our preliminary work on this topic examines mobility profiles for each of a number of subgroups taken separately (with subgroup membership defined by status in the base-year): see Jenkins and Van Kerm (2008). We find that income growth was especially pro-poor among family types concentrated at the bottom, notably families with children and pensioners - patterns that are consistent with the Labour government's aims to reduce pensioner and child poverty - but the small size of the subgroups weakens the statistical significance of these analyses.

\section{References}

Bennett, F. and Millar, J. (2005). Making work pay? Benefits, 13:28-33.

Böheim, R. and Jenkins, S. P. (2006). A comparison of current and annual measures of 
income in the British Household Panel Survey. Journal of Official Statistics, 22(4):733758.

Bourguignon, F. (2010). Non-anonymous growth incidence curves, income mobility and social welfare dominance: a theoretical framework with an application to the global economy. G-MonD Working Paper 14, Paris School of Economics, France. Downloadable from http://www.parisschoolofeconomics.eu/IMG/ pdf/WP14-GmonD-NaGrowthGlob-march10.pdf.

Brewer, M. and Shephard, A. (2004). Has Labour Made Work Pay? Joseph Rowntree Foundation, York. Downloadable from http://www.jrf.org.uk/bookshop/eBooks/ 1859352626 .pdf.

Cameron, A. C. and Trivedi, P. K. (2005). Microeconometrics: Methods and Applications. Cambridge University Press, Cambridge.

Chakravarty, S. R. (1984). Normative indices for measuring social mobility. Economics Letters, 15(1-2):175-180.

Checchi, D. and Dardanoni, V. (2003). Mobility comparisons: does using different measures matter? In Research on Economic Inequality, volume 9, pages 113-145. JAI Press, Stamford CT.

Chen, W.-H. (2009). Cross-national differences in income mobility: evidence from Canada, the United States, Great Britain and Germany. Review of Income and Wealth, $55(1): 75-100$.

Cleveland, W. S. (1979). Robust locally weighted regression and smoothing scatterplots. Journal of the American Statistical Association, 74(368):829-836.

Department for Work and Pensions (2008). Households Below Average Income. An analysis of the income distribution 1994/95-2006/0\%. Department for Work and Pensions, London. Downloadable from http://www.dwp.gov.uk/asd/hbai/hbai2007/contents. asp.

Donaldson, D. and Weymark, J. A. (1980). A single-parameter generalization of the Gini indices of inequality. Journal of Economic Theory, 22(1):67-86.

Dragoset, L. M. and Fields, G. S. (2006). U.S. earnings mobility: comparing surveybased and administrative-based estimates. ECINEQ Working Paper 2006-55, Society for the Study of Economic Inequality, Milan. Downloadable from http://www. ecineq. org/milano/WP/ECINEQ2006-55.pdf. 
Essama-Nssah, B. and Lambert, P. J. (2009). Measuring pro-poorness: a unifying approach with new results. Review of Income and Wealth, 55(3):752-778.

Fan, J. and Gijbels, I. (1996). Local Polynomial Modelling and its Applications. Chapman and Hall, London.

Fields, G. S. (2006). The many facets of economic mobility. In McGillivray, M., editor, Inequality, Poverty, and Well-Being, chapter 6, pages 123-142. Palgrave Macmillan, Houndsmills, Basingstoke.

Fields, G. S., Cichello, P. L., Freije, S., Menéndez, M., and Newhouse, D. (2003). For richer or for poorer? Evidence from Indonesia, South Africa, Spain, and Venezuela. Journal of Economic Inequality, 1(1):67-99.

Fields, G. S., Leary, J. B., and Ok, E. A. (2002). Stochastic dominance in mobility analysis. Economics Letters, 75(3):333-339.

Fields, G. S. and Ok, E. A. (1999a). The measurement of income mobility: an introduction to the literature. In Silber, J., editor, Handbook of Income Inequality Measurement, chapter 19, pages 557-596. Kluwer, Deventer.

Fields, G. S. and Ok, E. A. (1999b). Measuring movement of incomes. Economica, $66(264): 455-471$.

Gottschalk, P. and Danziger, S. (2001). Income mobility and exits from poverty of American children. In Bradbury, B., Jenkins, S. P., and Micklewright, J., editors, The Dynamics of Child Poverty in Industrialised Countries, chapter 5, pages 135-153. Cambridge University Press, Cambridge MA.

Gottschalk, P. and Huynh, M. (2010). Are earnings inequality and mobility overstated? The impact of non-classical measurement error. Review of Economics and Statistics, 92(2):302-315.

Gottschalk, P. and Moffitt, R. (2009). The rising instability of U.S. earnings. Journal of Economic Perspectives, 23(4):3-24.

Grimm, M. (2007). Removing the anonymity axiom in assessing pro-poor growth. Journal of Economic Inequality, 5(2):179-197.

Gruen, C. and Klasen, S. (2008). Growth, inequality, and welfare: comparisons across space and time. Oxford Economic Papers, 60(2):212-236. 
Jenkins, S. P. (1997). Trends in real income in Britain: a microeconomic analysis. Empirical Economics, 22(4):483-500.

Jenkins, S. P. (2010). The British Household Panel Survey and its income data. ISER Working Paper 2010-33, Institute for Social and Economic Research, University of Essex, Colchester, UK. Downloadable from http://www.iser.essex.ac.uk/pubs/ workpaps/pdf/2010-33.pdf.

Jenkins, S. P. (2011). Changing Fortunes: Income Mobility and Poverty Dynamics in Britain. Oxford University Press, Oxford. Forthcoming.

Jenkins, S. P. and Van Kerm, P. (2006). Trends in income inequality, pro-poor income growth and income mobility. Oxford Economic Papers, 58(3):531-548.

Jenkins, S. P. and Van Kerm, P. (2008). Has income growth in Britain become more pro-poor? Paper presented at the 30th General Conference of the International Association for Research on Income and Wealth, August 24-30 2008, Portoroz, Slovenia. Downloadable from http://www.iariw.org/papers/2008/vankerm.pdf.

Jenkins, S. P. and Van Kerm, P. (2009). The measurement of economic inequality. In Salverda, W., Nolan, B., and Smeeding, T. M., editors, The Oxford Handbook on Economic Inequality, chapter 3, pages 40-67. Oxford University Press, Oxford.

Joyce, R., Muriel, A., Phillips, D., and Sibieta, L. (2010). Poverty and inequality in the UK: 2010. IFS Commentary 116, Institute for Fiscal Studies, London. Downloadable from http://www.ifs.org.uk/comms/comm116.pdf.

Levy, H. and Jenkins, S. P. (2008). Documentation for Derived Current and Annual Net Household Income Variables, BHPS waves 1-16. Institute for Social and Economic Research, University of Essex, UK. Downloadable from http://www.esds.ac.uk/search/ indexSearch. asp?ct=xmlSn\&q1=3909.

Maasoumi, E. (1998). On mobility. In Ullah, A. and Giles, D. E. A., editors, Handbook of Applied Economic Statistics, chapter 5, pages 119-175. Marcel Dekker, New York.

Maasoumi, E. and Heshmati, A. (2000). Stochastic dominance amongst Swedish income distributions. Econometric Reviews, 19(3):287-320.

Mahalanobis, P. C. (1960). A method of fractile graphical analysis. Econometrica, 28(2):325-351.

Pen, J. (1971). Income Distribution. Penguin Books, Harmondsworth, Middlesex. 
Press, W. H., Teukolsky, S. A., Vetterling, W. T., and Flannery, B. P. (2007). Numerical Recipes: the Art of Scientific Computing. Cambridge University Press, New York, 3rd edition.

Ravallion, M. and Chen, S. (2003). Measuring pro-poor growth. Economics Letters, 78(1):93-99.

Saigo, H., Shao, J., and Sitter, R. R. (2001). A repeated half-sample bootstrap and balanced repeated replications for randomly imputed data. Survey Methodology, 27(2):189-196.

Saposnik, R. (1981). Rank-dominance in income distributions. Public Choice, 36(1):147151.

Sefton, T., Hills, J., and Sutherland, H. (2009). Poverty, inequality and redistribution. In Hills, J., Sefton, T., and Stewart, K., editors, Towards a More Equal Society? Poverty, Inequality and Policy since 1997, chapter 2, pages 21-45. Policy Press, Bristol, UK.

Sefton, T. and Sutherland, H. (2005). Inequality and poverty under New Labour. In Hills, J. and Stewart, K., editors, A More Equal Society, chapter 11, pages 231-249. Policy Press, Bristol, UK.

Sen, B. (2005). Estimation and comparison of fractile graphs using kernel smoothing techniques. Sankhyã, The Indian Journal of Statistics, 67(2):305-334.

Shorrocks, A. F. (1983). Ranking income distributions. Economica, 50(197):3-17.

Son, H. H. (2004). A note on pro-poor growth. Economics Letters, 82(3):307-314.

Van Kerm, P. (2006). Comparisons of income mobility profiles. IRISS Working Paper 2006-03, CEPS/INSTEAD, Differdange, Luxembourg. Downloadable from http:// www.ceps.lu/iriss/documents/irisswp58.pdf.

Van Kerm, P. (2009). Income mobility profiles. Economics Letters, 102(2):93-95.

Yitzhaki, S. (1983). On an extension of the Gini inequality index. International Economic Review, 24(3):617-628. 


\section{Appendix A Estimates with alternative approaches to measurement error}

\section{Appendix A.1 Using lead and lag income to 'instrument' current- year ranks}

(a) Absolute income mobility profiles

(b) Cumulative absolute income mobility profiles
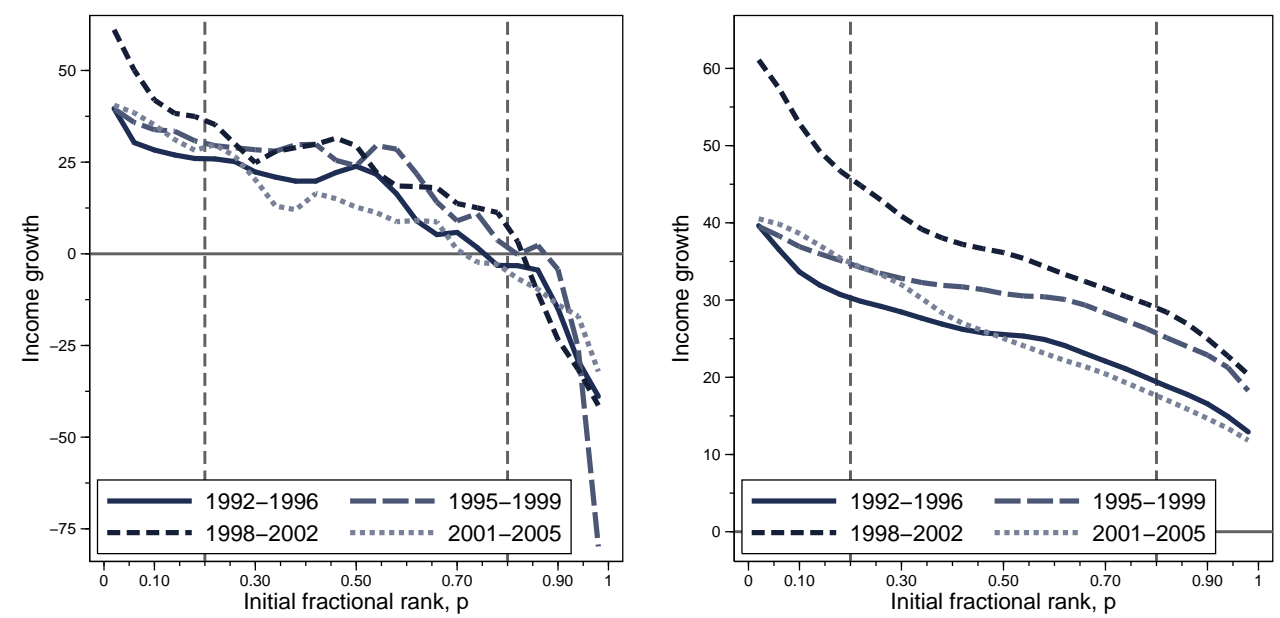

(c) Relative income mobility profiles

(d) Cumulative relative income mobility
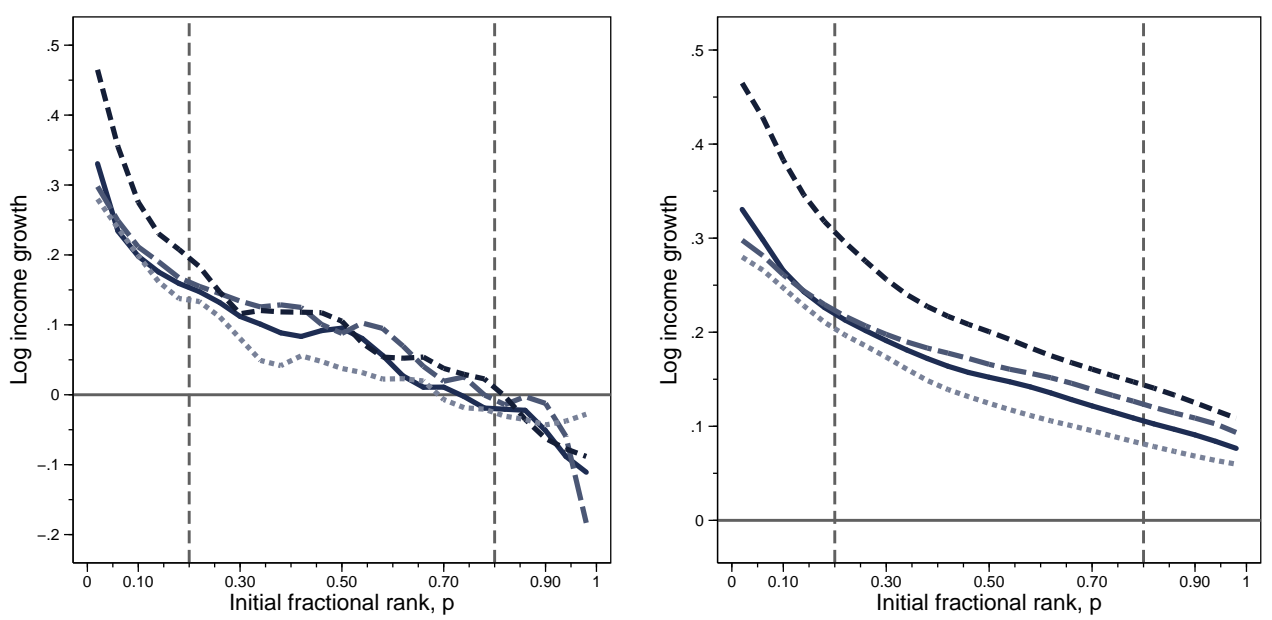

Figure A1. Income mobility profiles with proxied base year rank and no three-year-average smoothing

Note. Top panel shows absolute growth (change in real income); bottom panel shows proportionate growth (change in log real income). 

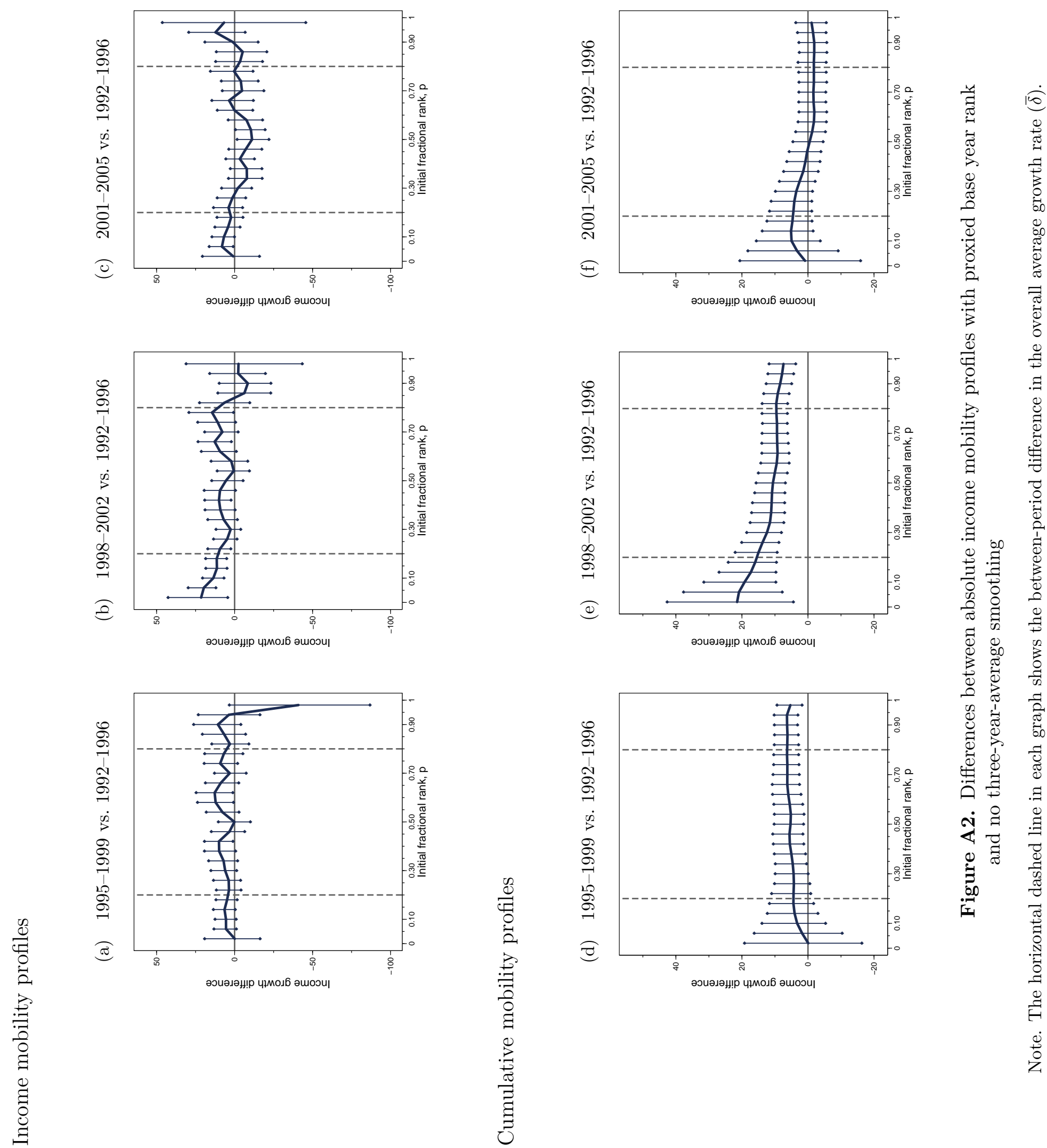

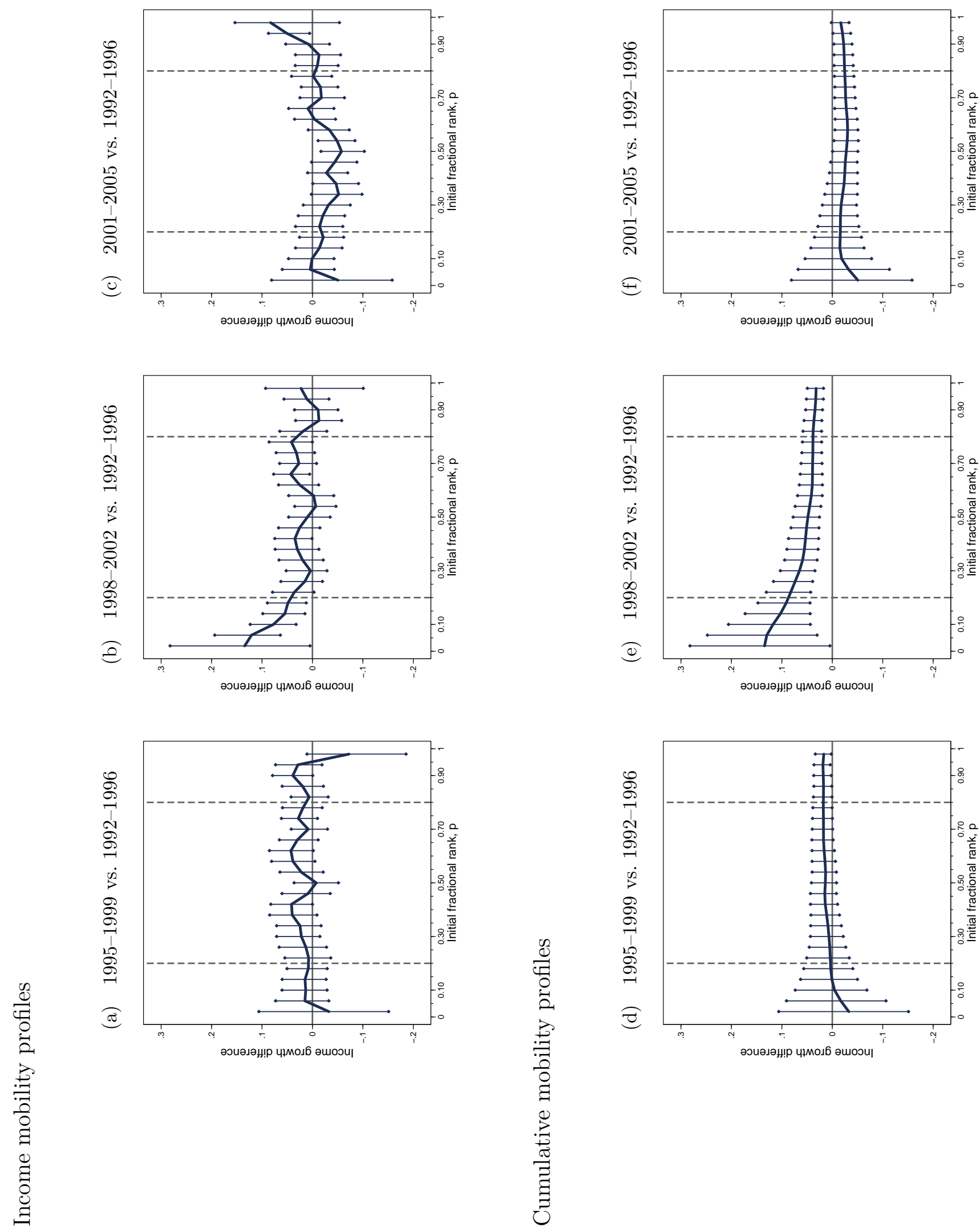


\section{Appendix A.2 Estimates for samples with better measured incomes: (i) dropping observations in households with a self-employed member}

(a) Absolute income mobility profiles

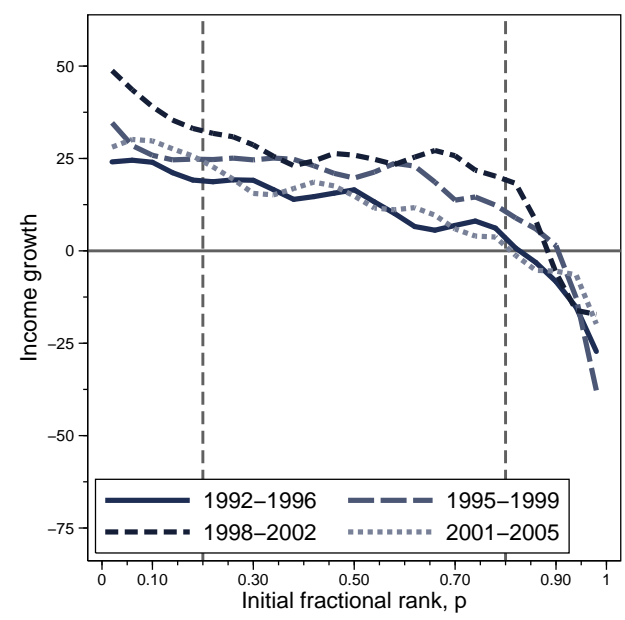

(c) Relative income mobility profiles

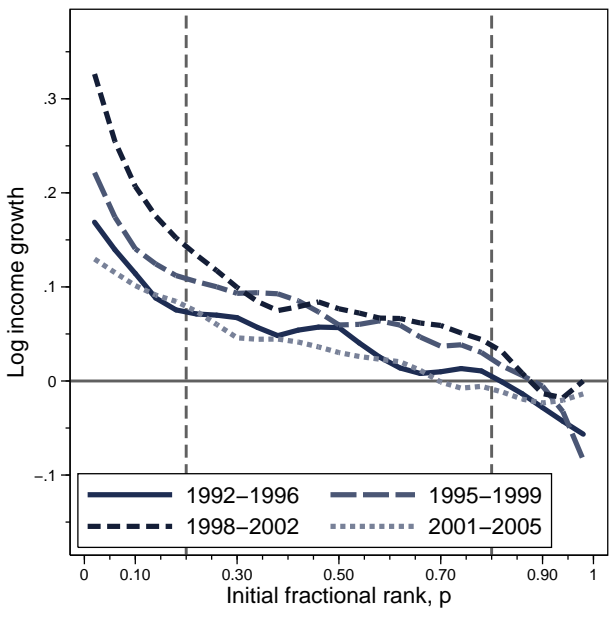

(b) Cumulative absolute income mobility profiles

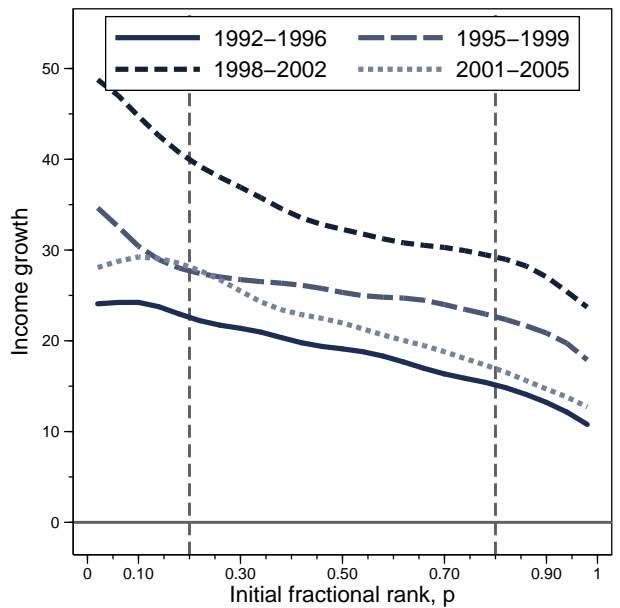

(d) Cumulative relative income mobility profiles

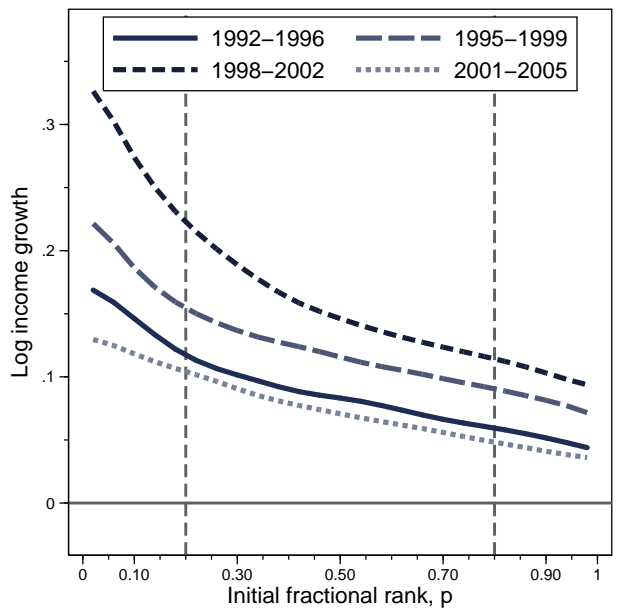

Figure A4. Income mobility profiles (no self-employed subsample)

Note. Top panel shows absolute growth (change in real income); bottom panel shows proportionate growth (change in log real income). 

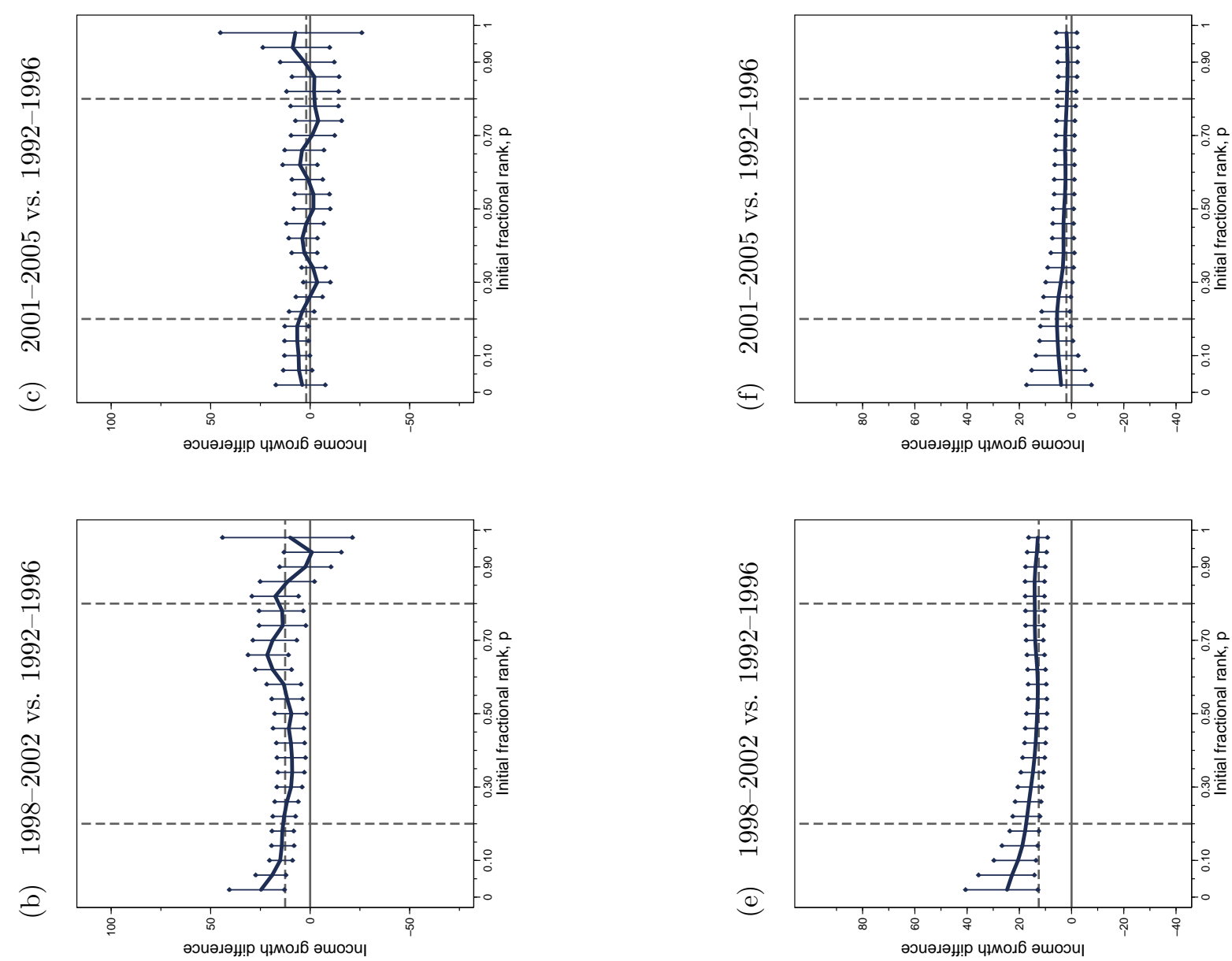

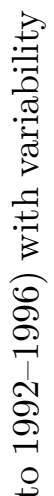

ஸि

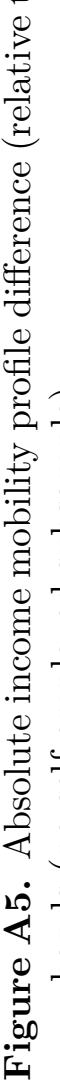

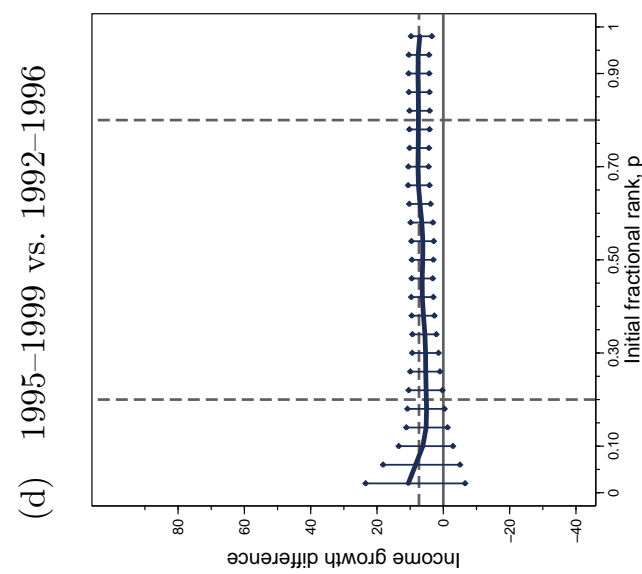

0
0
0
0
0
0
0
0
0
0
0
0
0
0

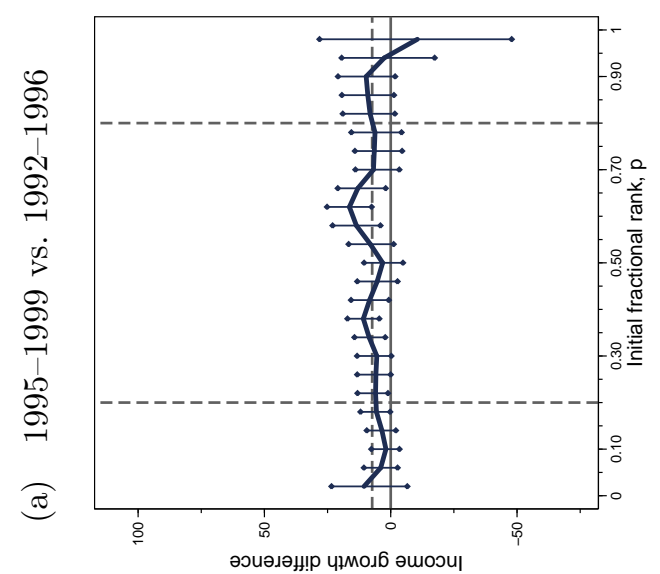

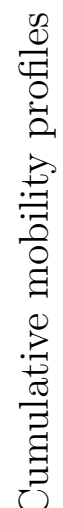

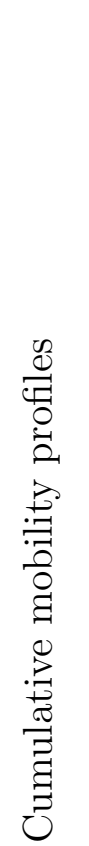



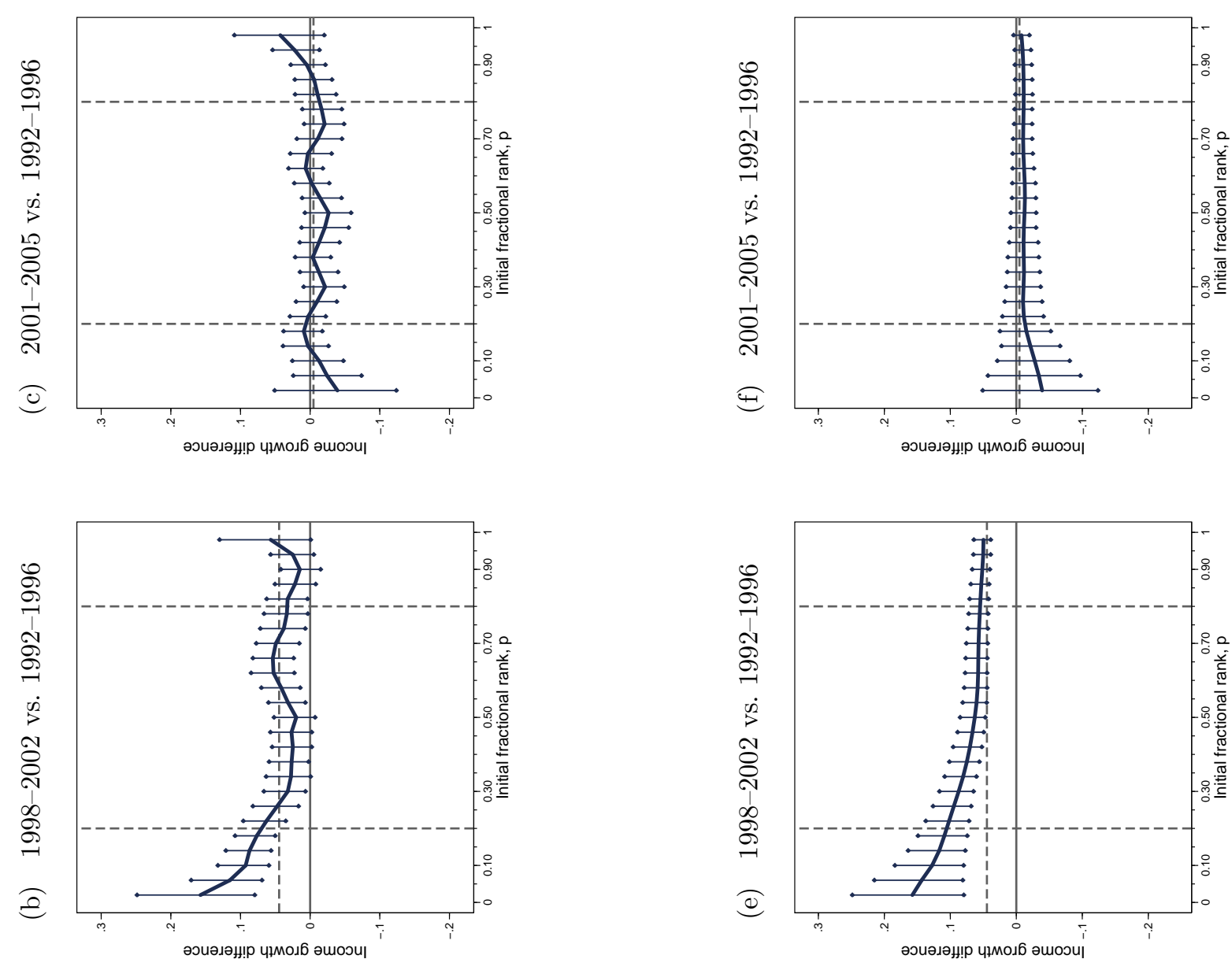

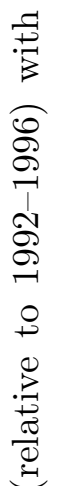

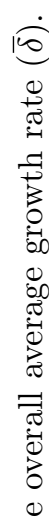

ن

幽

웅

है छ

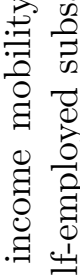

唨
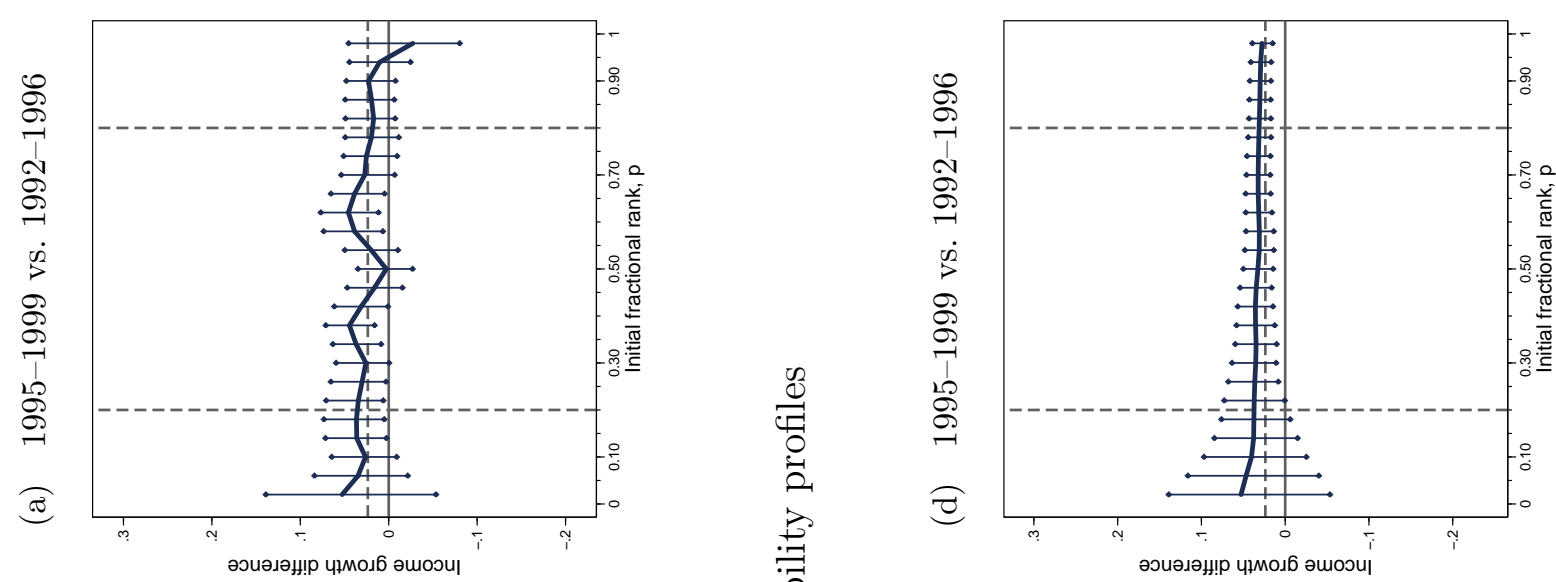

告

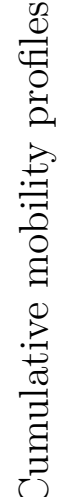

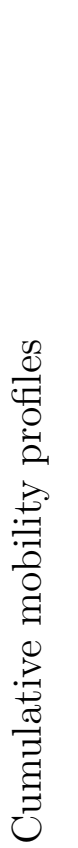




\section{Appendix A.3 Estimates for samples with better measured incomes: (ii) dropping observations with imputed income values}

(a) Absolute income mobility profiles

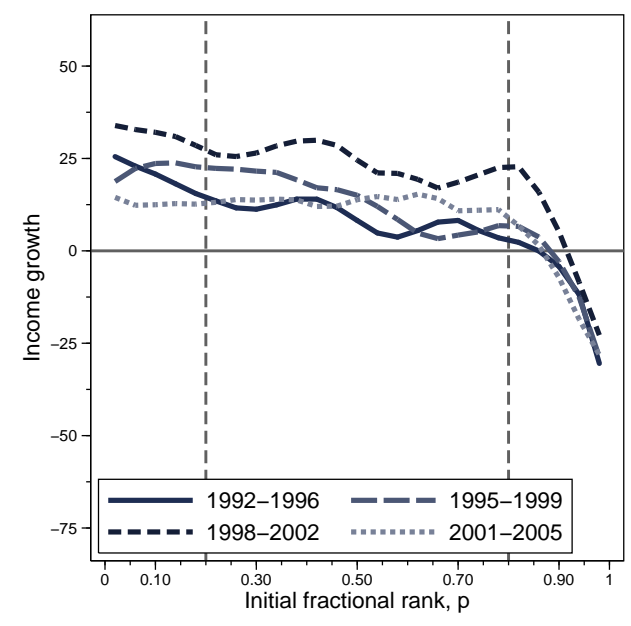

(c) Relative income mobility profiles

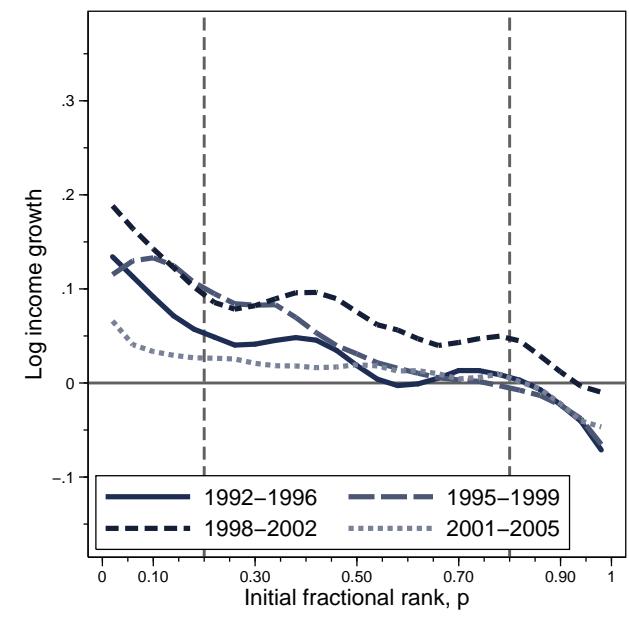

(b) Cumulative absolute income mobility profiles

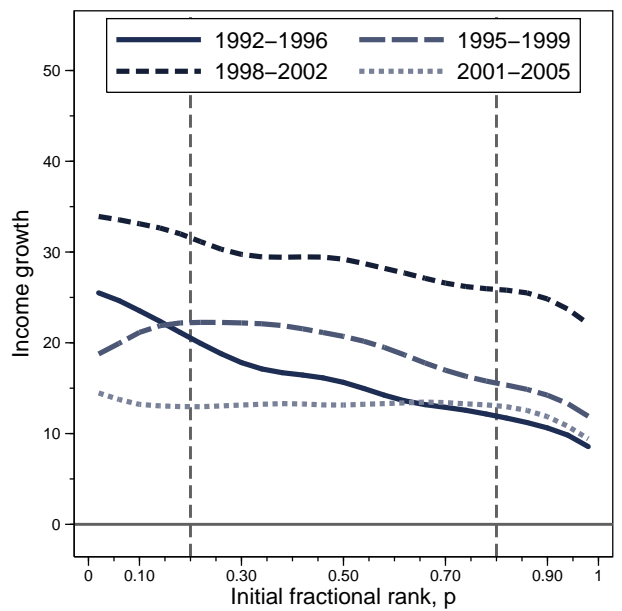

(d) Cumulative relative income mobility profiles

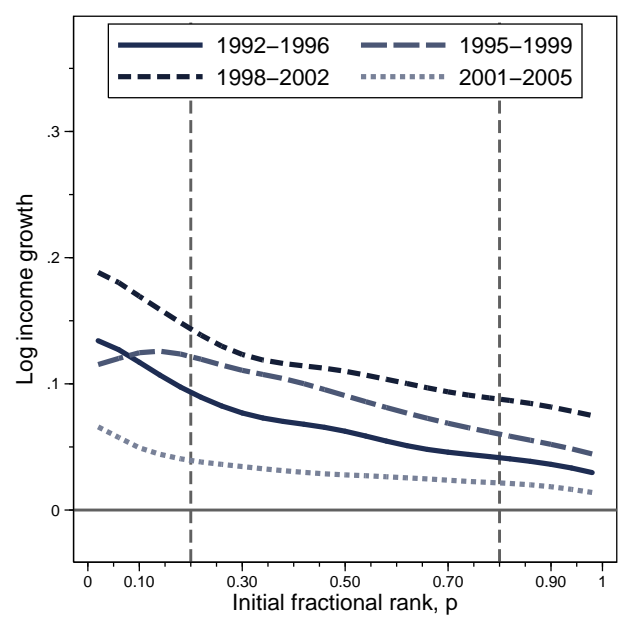

Figure A7. Income mobility profiles (no imputed income subsample)

Note. Top panel shows absolute growth (change in real income); bottom panel shows proportionate growth (change in log real income). 

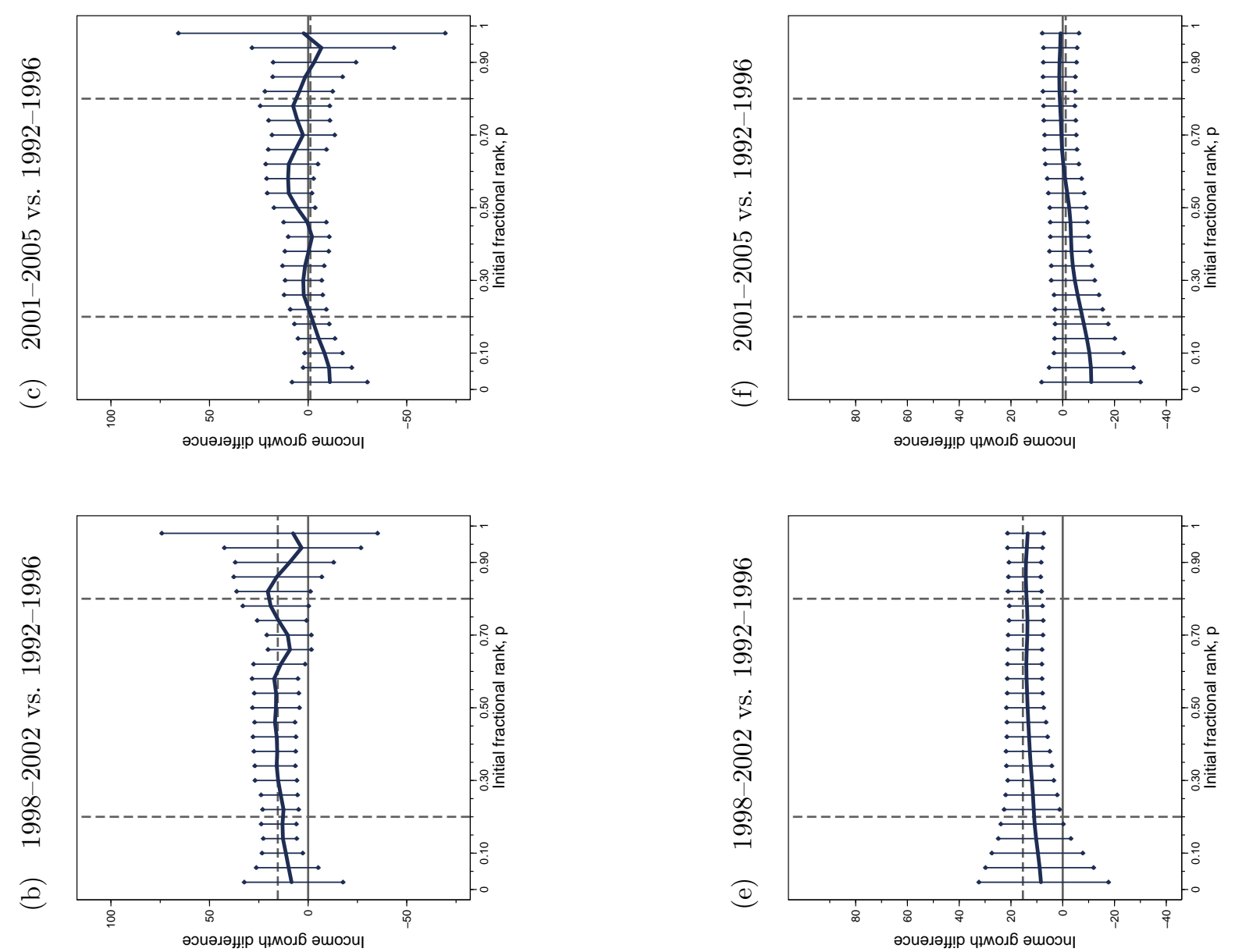

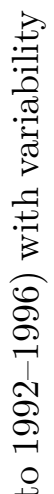

ஸि

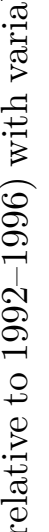

䒕

索

8

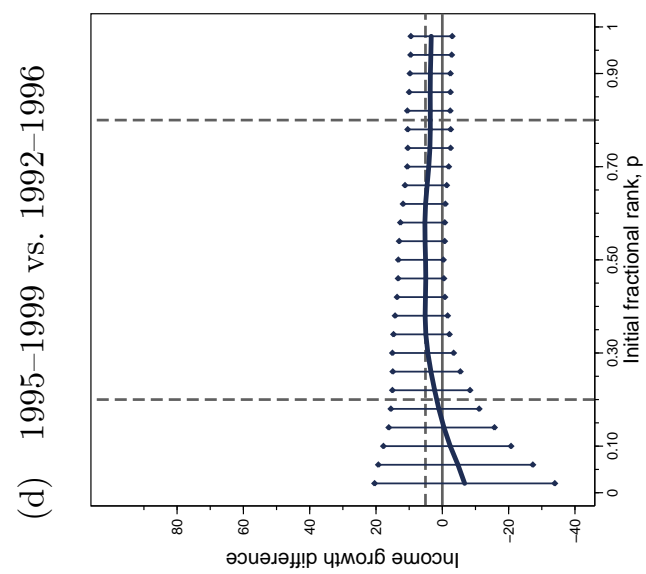



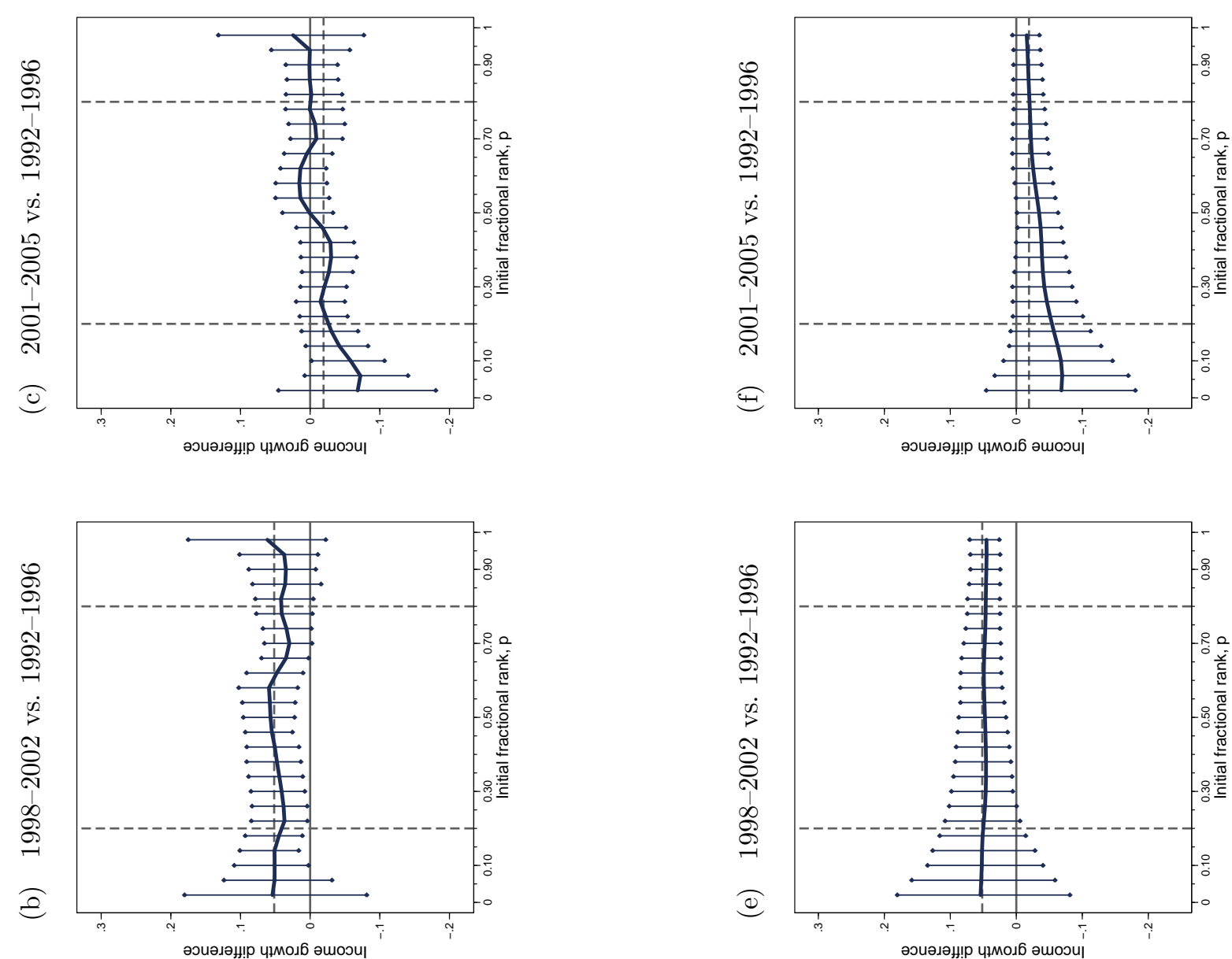

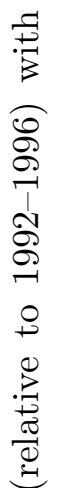

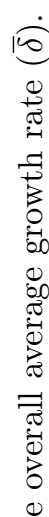

ن

式

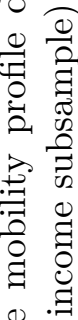

范
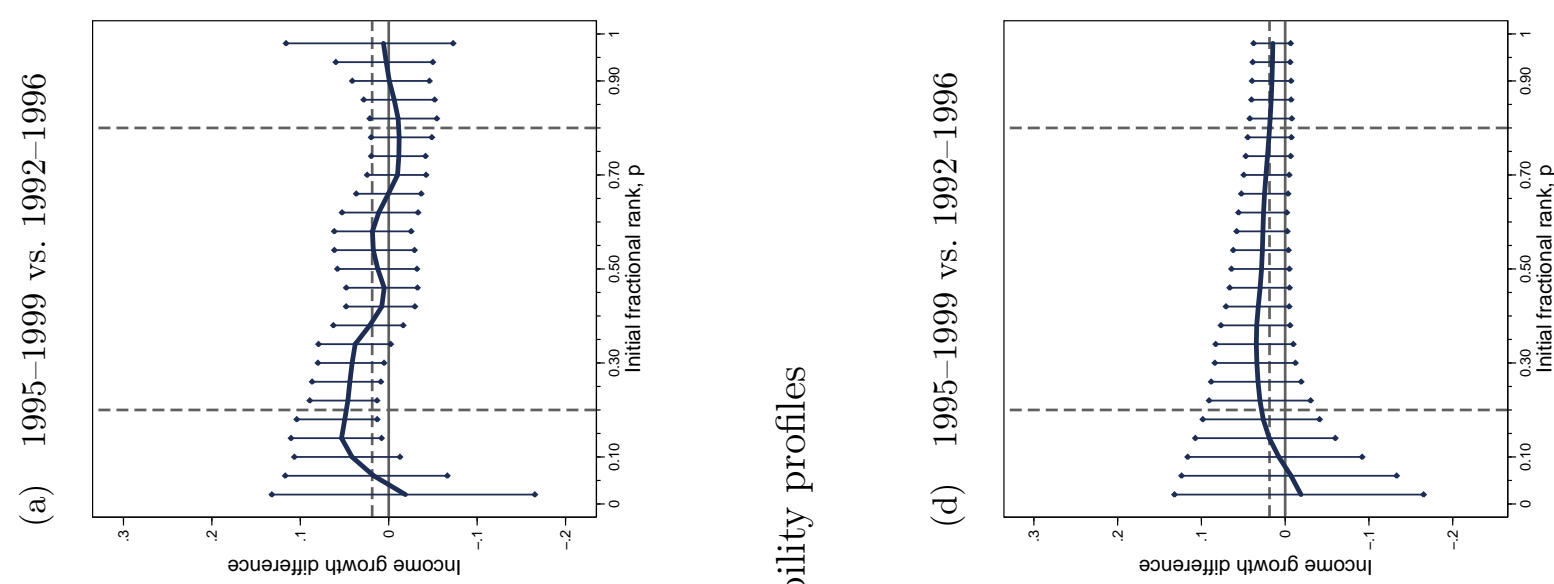

㐘

ฮ

әэนวләнцр чІмох6 әшоэи 
Appendix B Age-standardized estimates 

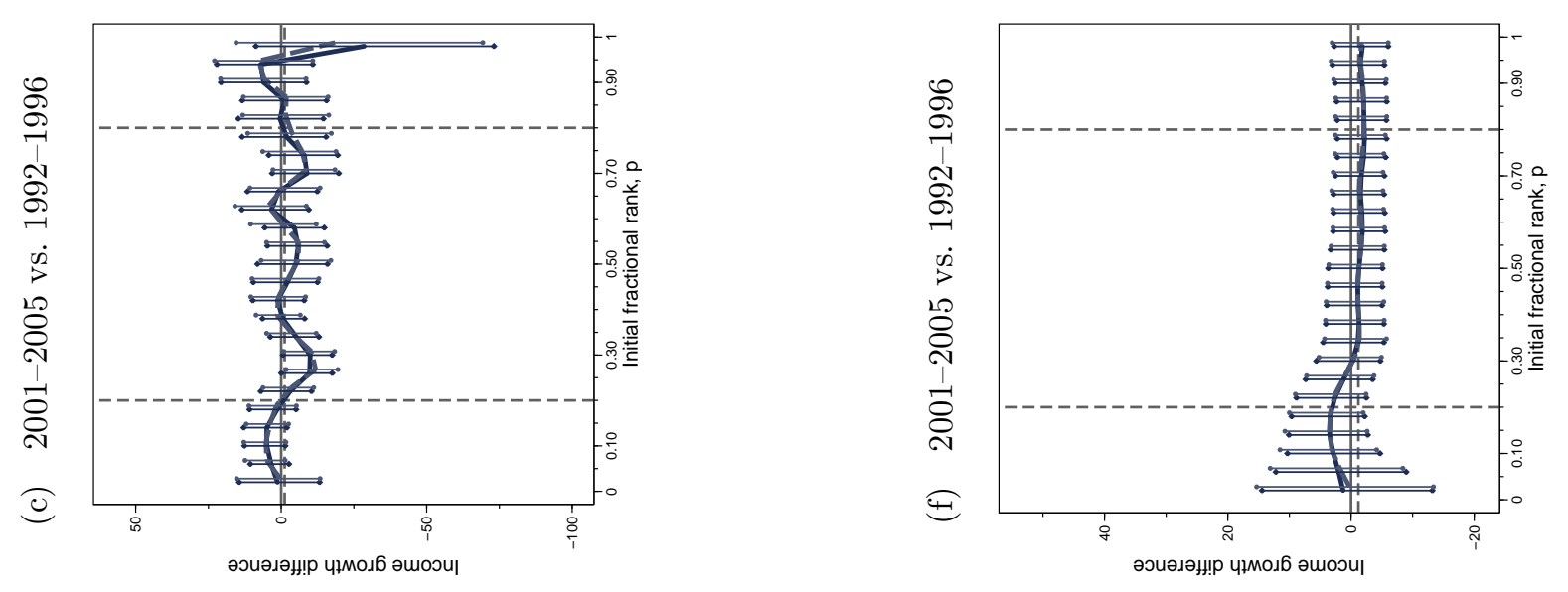

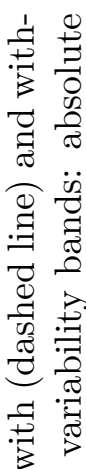
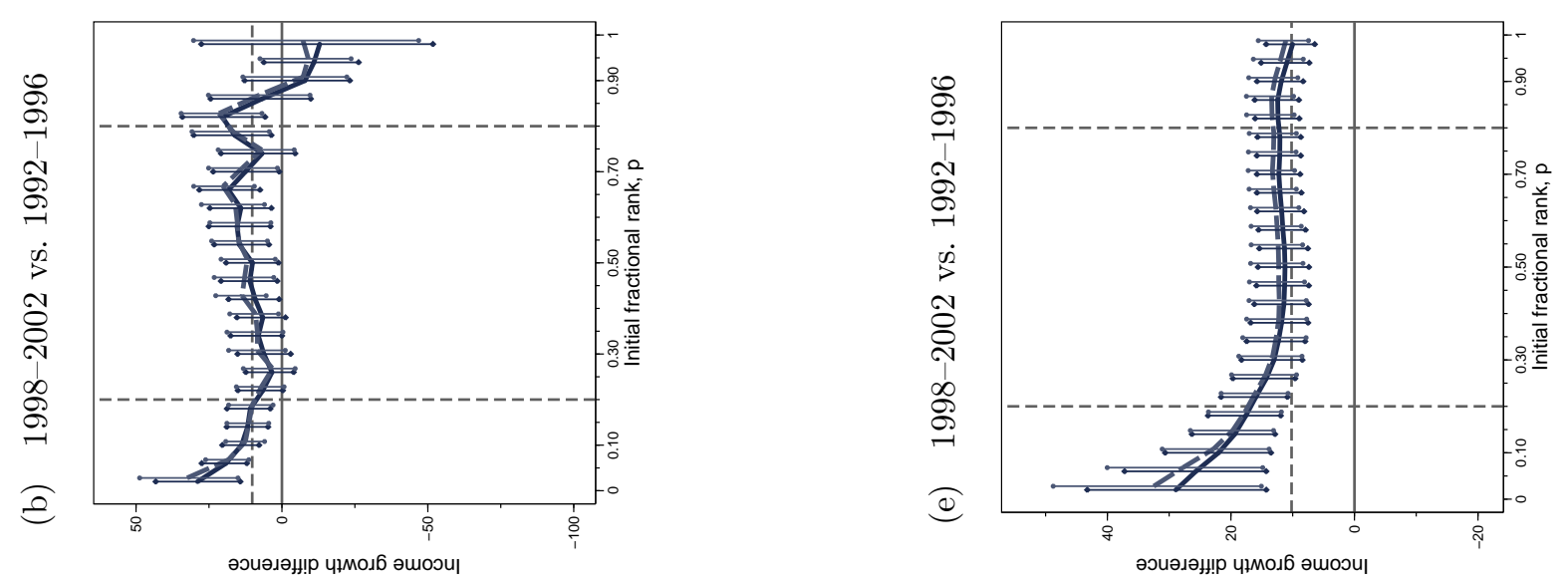

ชิㅇำ

$\frac{9}{5}$

票

ริ

ब

$\stackrel{\circ}{\circ}$

○)

焉 $\frac{0}{3}$

$\stackrel{4}{4}$

造

范

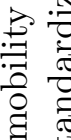
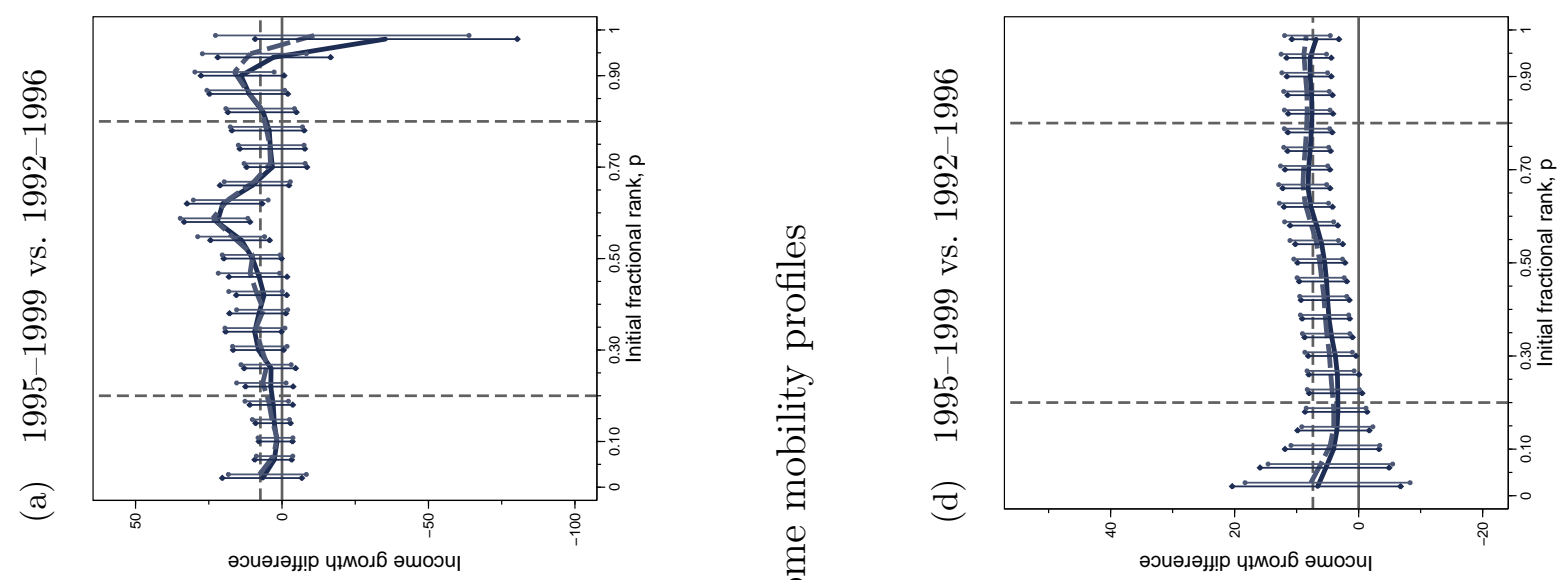

$\exists$

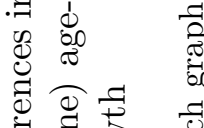

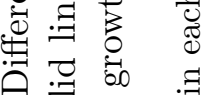

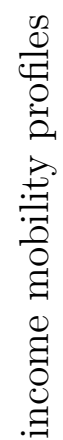

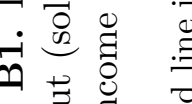

․․․

0
0
0
0
0
0
0
0
0
0
0
0
0
0
0
0

: 

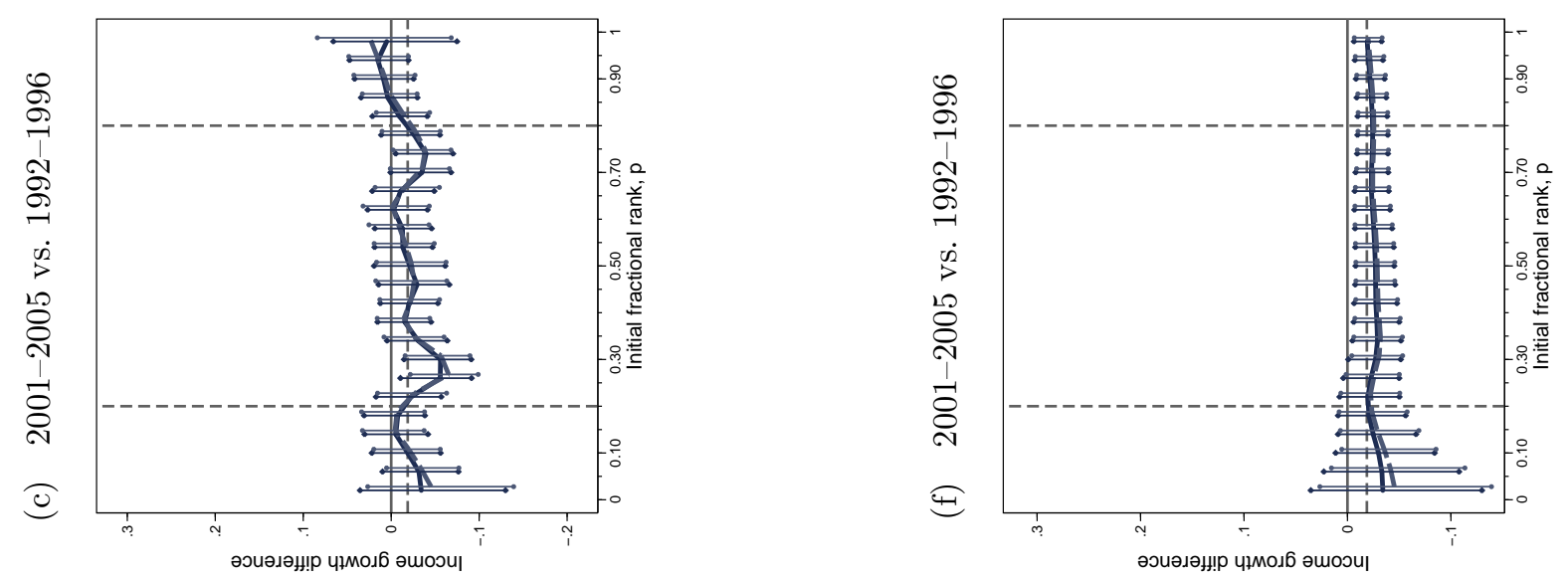

它

(0)

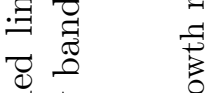

ज की

ت्]

돌

$3>$ \%

ㅇํㅇ

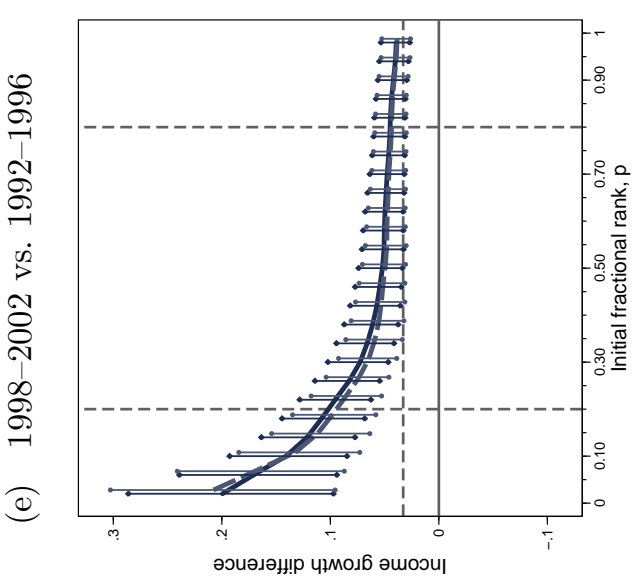

o

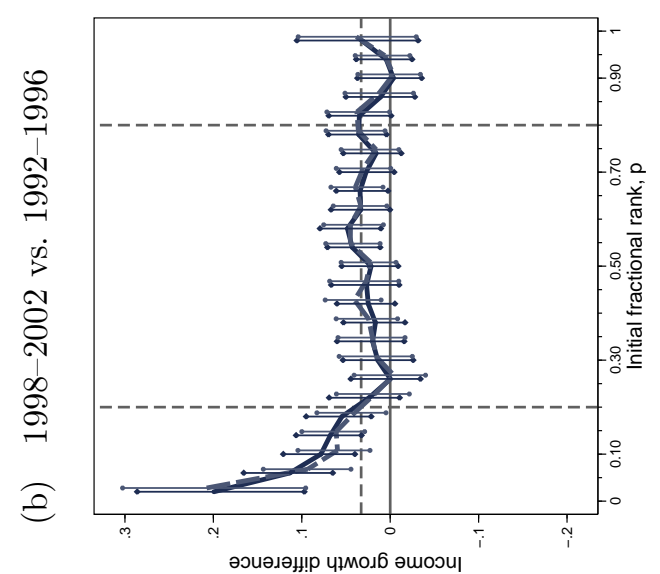

굘

g

की

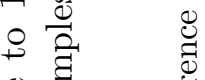

()

弯

$D_{0}^{\infty}$ क

\& न

등

ㄴ. 고

ㄱ.

泀苛

变

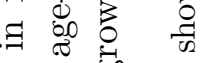
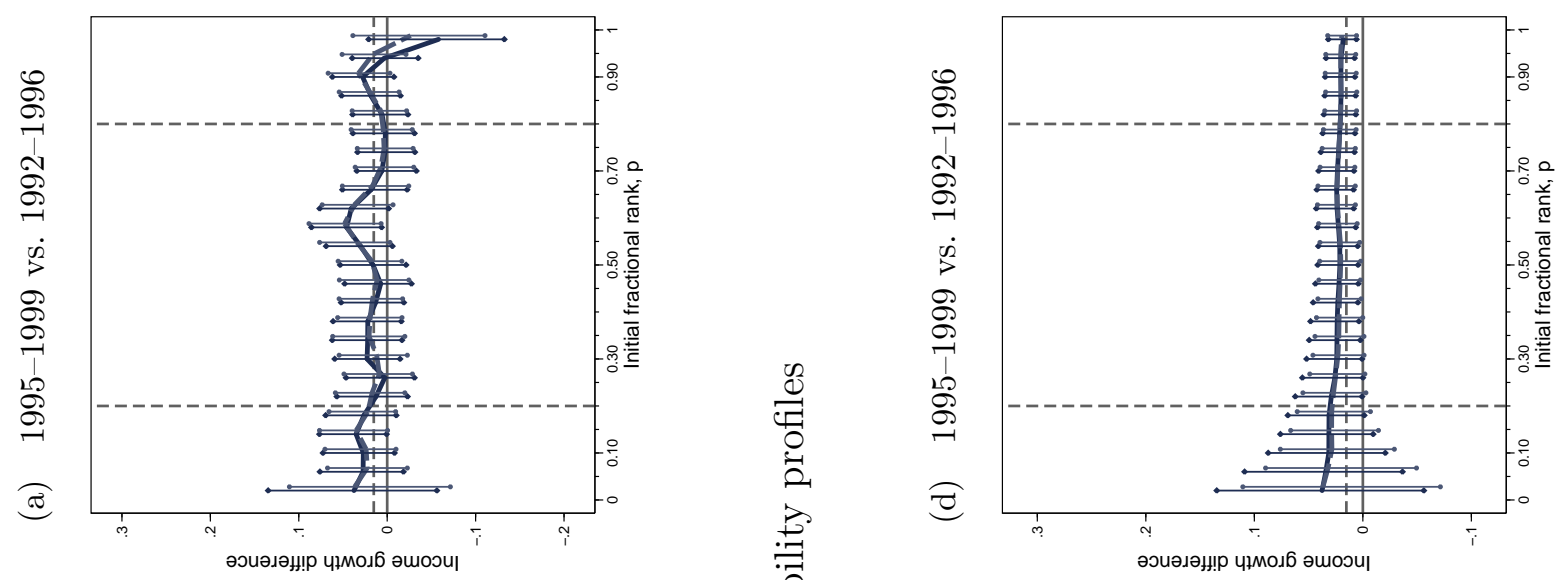

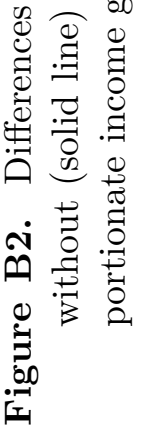

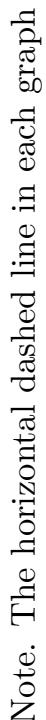

